\title{
Global Existence of Solutions to a Parabolic-Elliptic System of Drift-Diffusion Type in $R^{2}$
}

\author{
By \\ Toshitaka Nagai and Takayoshi Ogawa \\ (Hiroshima University and Tohoku University, Japan)
}

\begin{abstract}
We consider the Cauchy problem of a parabolic-elliptic system in $\boldsymbol{R}^{2}$, which appears in various fields in physics and biology. Under a mild restriction on the initial data, we show the global existence of nonnegative solutions to the Cauchy problem for both the subcritical and the critical cases, that is, the total mass of the solution is less than or equal to $8 \pi$.

Key Words and Phrases. Interaction particle, Gravitational model, Chemotaxis model, Global existence, Subcritical case, Two dimensions.

2010 Mathematics Subject Classification Numbers. Primary 35K15, 35K55, 35Q60; Secondary 78A35.
\end{abstract}

\section{Introduction}

We consider the following Cauchy problem of the parabolic-elliptic system in two space dimensions:

$$
\begin{cases}\partial_{t} u-\Delta u+\nabla \cdot(u \nabla \psi)=0, & t>0, x \in \boldsymbol{R}^{2}, \\ -\Delta \psi=u, & t>0, x \in \boldsymbol{R}^{2}, \\ u(0, x)=u_{0}(x), & x \in \boldsymbol{R}^{2},\end{cases}
$$

where the unknown function $u=u(t, x): \boldsymbol{R}_{+} \times \boldsymbol{R}^{2} \rightarrow \boldsymbol{R}$ denotes the density of the particles and $\psi=\psi(t, x): \boldsymbol{R}_{+} \times \boldsymbol{R}^{2} \rightarrow \boldsymbol{R}$ denotes the potential induced by the particle density and $u_{0}=u_{0}(x)$ is a nonnegative initial data. This system appears in various fields of physics and biology. One of the important model described by (1.1) is the movement of the gravitational particles in astronomy. The above system is also connected to a simplest equation of the semiconductor devise simulation (cf. Mock [41]) and a model of randam walk Patlak [55]. The semi-conductor devise model has a stabler sign of the nonlinearity that makes the system admits the large data global solutions.

We also mention that the semi-linear type of the equation is known as a model of chemotaxis called as the Keller-Segel system. Namely

$$
\begin{cases}\partial_{t} u-\Delta u+\nabla \cdot(u \nabla \psi)=0, & t>0, x \in \boldsymbol{R}^{n}, \\ \kappa \partial_{t} \psi-\Delta \psi+\lambda \psi=u, & t>0, x \in \boldsymbol{R}^{n}\end{cases}
$$


where $\kappa \geq 0, \lambda>0$, and $u \geq 0$ denotes the density of microorganisms and $\psi$ the concentration of a chemoattractant secreted by themselves. The system (1.1) is reduced from the original parabolic system (1.2) with $\kappa>0$ due to Keller-Segel [31] (see also [18]) under the condition that the chemical attraction is governed under large diffusive coefficient. It has been studied in detail for the behavior of the system by many authors Biler [4], Biler-Nadzieja [9], GajewskiZacharias [19], Herrero-Velázquez [26], Horstmann-Wang [28], Jäger-Luckhaus [29], Nagai [42], Nagai-Senba-Yoshida [48], Senba-Suzuki [58] and reference therein. Related results for chemotaxis models, see [25, 43, 47, 59], and for models of self-interacting particles, see [4, 6, 7, 13, 35, 52, 53, 64]. Many of these results for chemotaxis models also can be found in Horstmann [27] and Suzuki [61].

In this paper, we consider the global existence of the solution to (1.1) under some proper condition on the initial data. Before going to the global existence, we mention the time local existence and uniqueness. It is indeed strongly related to the results on the Navier-Stokes system in $\boldsymbol{R}^{2}$. The existence of the time local solution is essentially obtained by the similar argument of the case for the vorticity system of two dimensional Navier-Stokes equation:

$$
\begin{cases}\partial_{t} \omega-\Delta \omega+\nabla \cdot(u \omega)=0, & t>0, x \in \boldsymbol{R}^{2}, \\ -\Delta u=\nabla^{\perp} \omega, & t>0, x \in \boldsymbol{R}^{2}, \\ \omega(0, x)=\operatorname{rot} u_{0}(x), & x \in \boldsymbol{R}^{2},\end{cases}
$$

where $u=\left(u_{1}(t, x), u_{2}(t, x)\right)$ denotes the velocity fields for the incompressible flow and $\omega=\omega(t, x)$ is the vorticity of the fluid given by $\omega=\operatorname{rot} u=\partial_{1} u_{2}-\partial_{2} u_{2}$. The existence and uniqueness of the time local solution is given by GigaMiyakawa-Osada [23], Giga-Miyakawa [22], Giga-Kambe [21], Kato [30], BenAlti [1], Brezis [15] (see also [20]). Following their arguments, we may obtain the time local existence of the solution in some proper space.

Let us define the solution of the two dimensional Poisson equation in (1.1) as

$$
\psi(t, x)=\frac{1}{2 \pi} \int_{\boldsymbol{R}^{2}} \log \frac{1}{|x-y|} u(t, y) d y
$$

We denote by $L^{p}\left(\boldsymbol{R}^{2}\right)$ the usual Lebesgue space with the norm $\|\cdot\|_{p}$ for $1 \leq p \leq \infty$ and by $W^{s, p}\left(\boldsymbol{R}^{2}\right)$ the Sobolev space with $s>0$ and $1 \leq p \leq \infty$. For the following proposition on time local solutions of (1.1), see [45].

Proposition 1.1 (local existence and uniqueness). Let $u_{0} \in L^{1}\left(\boldsymbol{R}^{2}\right)$ satisfy $u_{0} \log (1+|x|) \in L^{1}\left(\boldsymbol{R}^{2}\right)$. Then there exists $T>0$ and a unique mild solution $(u, \psi)$ of $(1.1)$ such that 
(1) $u$ is in $C\left([0, T) ; L^{1}\left(\boldsymbol{R}^{2}\right)\right) \cap C\left((0, T) ; L^{4 / 3}\left(\boldsymbol{R}^{2}\right)\right)$ under the conditions

$$
\sup _{0<t<T} t^{1 / 4}\|u(t)\|_{4 / 3}<\infty, \quad \sup _{0<t<T}\|u(t) \log (1+|x|)\|_{1}<\infty,
$$

(2) $(u, \psi)$, where $\psi$ is defined by (1.4), satisfies the following integral equation

$$
u(t)=e^{t \Delta} u_{0}-\int_{0}^{t} \nabla \cdot e^{(t-s) \Delta}(u(s) \nabla \psi(s)) d s, \quad 0<t<T,
$$

where $e^{t \Delta}$ is the heat semigroup defined by

$$
\left(e^{t \Delta} f\right)(x)=\int_{\boldsymbol{R}^{2}} \frac{1}{4 \pi t} e^{-|x-y|^{2} /(4 t)} f(y) d y .
$$

Moreover, $(u, \psi)$ satisfies the following:

(1) Both $u$ and $\psi$ are smooth and $(u, \psi)$ is a classical solution of (1.1).

(2) If $u_{0}(x) \geq 0, u_{0} \neq \equiv$ then $u(t, x)>0$ for all $t \in(0, T)$ and $x \in \boldsymbol{R}^{2}$.

(3) The total mass is conserved, namely $\int_{\boldsymbol{R}^{2}} u(t) d x=\int_{\boldsymbol{R}^{2}} u_{0} d x$ for $0<t<T$.

Remark. For $(u, \psi)$ in Proposition 1.1, $\psi(t)$ is well-defined because of $u(t) \log (1+|x|) \in L^{1}\left(\boldsymbol{R}^{2}\right)$, and $u$ satisfies $\partial_{t}^{k} \partial_{x}^{\ell} u \in L^{p}\left(\boldsymbol{R}^{2}\right)$ for all $k \geq 0, \ell \geq 0$ and all $1<p \leq \infty$ (see [45]).

The existence of the time local solution is also discussed in KurokibaOgawa [34] in the weighted $L^{2}$ space such as

$$
\int_{\boldsymbol{R}^{2}}|x|^{2 s}|u(t)|^{2} d x<\infty
$$

for $s>1$, that is included in $L^{1}\left(\boldsymbol{R}^{2}\right)$ and [36] for $s=1$. Note that the uniqueness up to the constant for the Poisson equation is rather subtle (cf. Benilan-Brezis-Crandall [2]). Under this weight condition, the solution of the Poisson equation is determined uniquely up to constant.

We now concentrate our attention on the global existence of the solution. For the positive large data, it is known that the solution does not exists globally in time (see, $[4,43,58,59,61])$. On the other hand, the global existence of the solution is obtained for the bounded domain $\Omega \subset \boldsymbol{R}^{2}$ under the smallness assumption on the initial mass

$$
\int_{\Omega} u_{0}(x) d x<8 \pi
$$

when the solution is nonnegative. The argument heavily depends on the use of the free energy functional

$$
F[u](t) \equiv \int_{\Omega} u(t) \log u(t) d x-\frac{1}{2}\|\nabla \psi(t)\|_{2}^{2}
$$


It is used that the free energy functional satisfies the following estimate

$$
F[u](t)+\int_{0}^{t} \int_{\Omega} u|\nabla \log u(t)-\nabla \psi(t)|^{2} d x d t \leq F\left[u_{0}\right]
$$

so that the free energy is monotone decreasing in time and if the data is small then one may obtain the a priori estimate for the higher regularity functional of the solution such as $u(t) \in L^{\infty}(0, T ; L \log L(\Omega))$. The standard parabolic regularity argument gives the boundedness of the solution $u$ and hence the global existence for (1.1) for bounded domain with 0 -Neumann boundary condition is obtained.

Similar argument is also valid for the chemotaxis model (1.2). Indeed, the system (1.1) also has analogous property in the structure. Formally we have that the free energy functional

$$
F[u](t) \equiv \int_{\boldsymbol{R}^{2}} u(t) \log u(t) d x-\frac{1}{2}\|\nabla \psi(t)\|_{2}^{2}-\frac{\lambda}{2}\|\psi(t)\|_{2}^{2}
$$

is bounded by the initial data. However the proof of the global existence for (1.1) is rather complicated since the behavior of the solution of (1.1) is different from the one in (1.2). Namely one can not expect the free energy functional holding by its original form and it is not straightforward to derive the a priori bound for the solution. The proof of the a priori estimate for $u$ in $L \log L\left(\boldsymbol{R}^{2}\right)$ space is formally very similar to the case of (1.2) by letting $\kappa=\lambda=0$. However, there is a significant difference of the nature of the solution of the above system (1.1) from the case of the bounded domain or the solution of (1.2) because of the behavior of $\psi$ at $|x| \rightarrow \infty$. If we discuss the solution of the first equation $u(t)$ in $L^{1}\left(\boldsymbol{R}^{2}\right)$, then applying the Gauss divergence theorem to the second equation, we see that for any $R^{\prime}>R>0$,

$$
\begin{aligned}
\|\nabla \psi(t)\|_{2}^{2} & \geq \int_{R}^{R^{\prime}} \int_{0}^{2 \pi}\left|\partial_{r} \psi(r, \omega)\right|^{2} r d \omega d r \\
& \geq \frac{1}{2 \pi} \int_{R}^{R^{\prime}}\left(\int_{0}^{2 \pi}\left(\partial_{r} \psi(r, \omega)\right) r d \omega\right)^{2} \frac{1}{r} d r \\
& =\frac{1}{2 \pi} \int_{R}^{R^{\prime}}\left(\int_{B_{r}} u(y) d y\right)^{2} \frac{1}{r} d r \geq \frac{1}{2 \pi}\left(\int_{B_{R}} u(y) d y\right)^{2} \log \frac{R^{\prime}}{R} \rightarrow \infty
\end{aligned}
$$

as $R^{\prime} \rightarrow \infty$. Hence as far as treating the positive solution for the first equation, we can not assume that $\nabla \psi(t) \in L^{2}\left(\boldsymbol{R}^{2}\right)$ for any time. To avoid this difficulty, one may introduce the modified form of the free energy functional

$$
H[u](t) \equiv \int_{\boldsymbol{R}^{2}} u(t) \log u(t) d x-\frac{1}{2} \int_{\boldsymbol{R}^{2}} u(t) \psi(t) d x,
$$


which is non-increasing in $t$ (cf. Blanchet-Dolbeault-Perthame [14]). $\quad H[u](t)$ is formally equivalent to the original functional $F[u](t)$ in (1.6) for the case $\lambda=0$ in view of the Poisson equation. However the justification of the equivalence is not quite straightforward and simple cut off argument does not seems to work well.

In this paper, we employ the following modified free energy functional

$$
H[u](t) \equiv \int_{\boldsymbol{R}^{2}}(1+u(t)) \log (1+u(t)) d x-\frac{1}{2} \int_{\boldsymbol{R}^{2}} u(t) \psi(t) d x .
$$

The modified entropy $\int_{\boldsymbol{R}^{2}}(1+u(t)) \log (1+u(t)) d x$ in (1.7) has the advantage of being nonnegative and has been used to get nonnegative global solutions for (1.2) with $\kappa>0$ by Nagai-Ogawa [46] in case of $\int_{\boldsymbol{R}^{2}} u_{0} d x<4 \pi$ and by Mizoguchi [40] in case of $\int_{\boldsymbol{R}^{2}} u_{0} d x<8 \pi$. The entropy $\int_{\boldsymbol{R}^{2}} u(t) \log u(t) d x$ has been used for (1.2) with $\kappa>0$ by Calvez-Corrias [17] in case of $\int_{\boldsymbol{R}^{2}} u_{0} d x<8 \pi$ and by Mizoguchi [40] in case of $\int_{\boldsymbol{R}^{2}} u_{0} d x=8 \pi$.

Our result on the global existence for (1.1) now reads as follows:

Theorem 1.2 (Global existence). Suppose that $u_{0} \in L^{1}\left(\boldsymbol{R}^{2}\right)$ is nonnegative and satisfies

$$
\int_{\boldsymbol{R}^{2}} u_{0} \log (1+|x|) d x<\infty
$$

Then under the condition

$$
\int_{R^{2}} u_{0} d x \leq 8 \pi
$$

the nonnegative solution $(u, \psi)$ to (1.1) in Proposition 1.1 exists globally in time. Moreover $(u, \psi)$ satisfies the properties mentioned in Proposition 1.1 for all $0<T<\infty$, and for any $0<t_{0}<T<\infty$,

$$
\int_{\boldsymbol{R}^{2}}(1+u(t)) \log (1+u(t)) d x-\frac{1}{2} \int_{\boldsymbol{R}^{2}} u(t) \psi(t) d x \leq C_{t_{0}, T}, \quad t \in\left[t_{0}, T\right] .
$$

In the subcritical case $M:=\int_{R^{2}} u_{0} d x<8 \pi$, the global existence of nonnegative weak solutions of (1.1) has been studied by Blanchet-DolbeaultPerthame [14] with the additional restrictions on the data;

$$
\begin{aligned}
& \left(1+|x|^{2}\right) u_{0} \in L^{1}\left(\boldsymbol{R}^{2}\right), \\
& \int_{\boldsymbol{R}^{2}} u_{0} \log \left(1+u_{0}\right) d x<\infty .
\end{aligned}
$$


Under these conditions, the negative part of the free energy is controlled by the second moment identity

$$
\int_{\boldsymbol{R}^{2}}|x|^{2} u(t) d x d x=\int_{\boldsymbol{R}^{2}}|x|^{2} u_{0} d x+4 M\left(1-\frac{M}{8 \pi}\right) t .
$$

Without additional weight restrictions on the nonnegative mild solution for $|x|$ large, Nagai [45] showed that the $L^{p}$-norm $(1 \leq p \leq \infty)$ of the solution is less than or equal to that of a radially symmetric nonnegative self-similar solution to (1.1) by using rearrangement techniques, which gives the global existence and decay estimates of the solution.

The critical mass for the existence of the global solutions is given by $M=8 \pi$. When the initial data have the critical mass $M=8 \pi$ there are some results known. See for instance $[8,56]$ for radially symmetric nonnegative solutions. Without symmetry assumptions on the solutions, Blanchet-CarrilloMasmoudi [13] proved that the nonnegative solutions with finite second moment exist globally in time and converge to a Dirac delta function as time goes to infinity. The system (1.1) for the critical mass case admits the following stationary solutions

$$
w(x ; b)=\frac{8 b}{\left(|x|^{2}+b\right)^{2}}, \quad b>0,
$$

which have no finite second moment. Blanchet-Carlen-Carrillo [11] have studied the global existence and long time behavior for the nonnegative solutions whose second moment is infinite but first moment is finite, by introducing the following functional

$$
H_{b}[u]=\int_{\boldsymbol{R}^{2}}(\sqrt{u(x)}-\sqrt{w(x ; b)})^{2}(w(x ; b))^{-1 / 2} d x,
$$

which comes from the critical fast diffusion equation in $\boldsymbol{R}^{2}$. See also LópezGómez-Nagai-Yamada [39] for global existence and large time behavior.

For the supercritical case $M>8 \pi$, under suitable assumption, the positive solution blows up in a finite time (see $[4,3,9,10,42,43]$ under the radially and non radially symmetric cases and [34] under non finite second moment case).

We emphasize that our result on the global existence of nonnegative solutions does not require a finite first moment of the solution and it holds true for all nonnegative initial data $u_{0} \in L^{1}\left(\boldsymbol{R}^{2}\right)$ with the least weighted condition $u_{0} \log (2+|x|) \in L^{1}\left(\boldsymbol{R}^{2}\right)$. In order to prove Theorem 1.2 , by virtue of Proposition 1.3 below (for the proof, see [45]) it suffices to prove the 
following: For a time local nonnegative solution $u$ over arbitrary time interval $[0, T)$

$$
\limsup _{t \rightarrow T} \int_{\boldsymbol{R}^{2}}(1+u(t)) \log (1+u(t)) d x<\infty .
$$

This is proved in Theorem 3.1 in Section 3.

Proposition 1.3. Let $T_{m}$ be the maximal existence time of the nonnegative solution $u$. If $T_{m}<\infty$, then

$$
\limsup _{t \rightarrow T_{m}} \int_{\boldsymbol{R}^{2}}(1+u(t)) \log (1+u(t)) d x=\infty .
$$

Remark. In $[13,14]$, under the assumptions $u_{0} \log u_{0}, u_{0}|x|^{2} \in L^{1}\left(\boldsymbol{R}^{2}\right)$ it was proved that if $T_{m}<\infty$, then

$$
\limsup _{t \rightarrow T_{m}} \int_{R^{2}} u(t) \log u(t) d x=\infty .
$$

However (1.13) and (1.14) are equivalent under the weaker assumption $u_{0} \log (2+|x|) \in L^{1}\left(\boldsymbol{R}^{2}\right)$ by virtue of Lemma 2.3 in Section 2 .

As we have mentioned above, the usual free energy functional $F(t)$ in $(1.5)$ is useful for the bounded domain $\Omega \subset \boldsymbol{R}^{2}$, however it is not suitable for the whole space case since the first and the second terms are not well behaved. Instead of that, to prove (1.12) we use the modified free energy functional $H(t)$ in (1.7). The key idea to show the global a priori bound is that we split the whole space into three pieces; a bounded ball (interior region), an exterior region and the annulus that connects the ball and the exterior region:

$$
\boldsymbol{R}^{2}=B_{R} \cup A_{R} \cup E_{R},
$$

where

$$
\begin{array}{ll}
B_{R}=\left\{x \in \boldsymbol{R}^{2} ;|x|<R\right\} ; & \text { interior region, } \\
A_{R}=\left\{x \in \boldsymbol{R}^{2} ; R / 2 \leq|x| \leq 2 R\right\} ; & \text { connecting annulus, } \\
E_{R}=\left\{x \in \boldsymbol{R}^{2} ; R<|x|\right\} ; & \text { exterior region. }
\end{array}
$$

The difficulty is that since the equation has the non-local interaction, such a localization is not quite possible and there remains a term involving the integral on the out side of each regions. Thanks to the special situation of the two space dimensions and the preferable behavior of the integral kernel of $(-\Delta)^{-1}$, it is controllable when we consider the estimate in the exterior region $E_{R}$ with sufficiently large $R>0$. Then it enable us to derive the a priori estimate in the exterior region (see Proposition 3.2 below). The estimate for the annulus $A_{R}$ follows from the estimate in $E_{R}$. Finally, we derive the interior estimate by 
using the critical type Sobolev inequality due to Brezis-Merle [16]. Namely, for a bounded domain $\Omega \subset \boldsymbol{R}^{2}$, Brezis-Merle showed that for a solution $v$ to the Poisson equation;

$$
\begin{cases}-\Delta v=f, & x \in \Omega, \\ v(x)=0, & x \in \partial \Omega,\end{cases}
$$

it holds that

$$
\int_{\Omega} e^{|v(x)|} d x \leq \frac{C d(\Omega)^{2}}{4 \pi-\|f\|_{1}},
$$

provided $f$ in $L^{1}(\Omega)$ and $\|f\|_{1}<4 \pi$, where $d(\Omega)$ is the diameter of $\Omega$ (see Lemma 2.7 below). With the aid of a priori estimates over the annulus $A_{R}$, we derive

$$
\sup _{0<t<T} \int_{B_{R}} u(t) d x<8 \pi
$$

and then, applying the Brezis-Merle inequality (1.16) we establish the global bound for the modified entropy over $B_{R}$ (see Proposition 3.16 below). This procedure makes us possible to obtain the time global a priori estimate for the modified entropy $\int_{R^{2}}(1+u) \log (1+u) d x$.

It is also worth to mention that the two dimensional case is both $L^{1}$ critical case and the Sobolev critical case. Those critical cases can be observed by regarding the quasilinear parabolic type system with the degenerate diffusion $-\Delta u^{\alpha}$ instead of $-\Delta u$ in the equation ( $\left.\mathrm{cf}[12,32,33,50,51,60,62]\right)$. When $\alpha=2-2 / n$ it is the $L^{1}$ critical case and the $L^{1}$ norm of the initial data can determine the large time behavior of the solution while if $\alpha=2-4 /(n+2)$ then the (dual) Sobolev critical norm $L^{2 n /(n+2)}$ of the data determines the large time behavior $([50,51])$. When $n=2$, those critical exponents $\alpha=2-2 / n$ and $\alpha=2-4 /(n+2)$ coincides and the semi-linear case is the doubly critical and the critical mass $8 \pi$ is the simultaneous threshold in the both critical cases. We also note that higher dimensional case for the system (1.1) is considered in $[3,37,54,57]$ and the solution blows up in a finite time in a weaker assumptions.

In the paper, we use a universal constant $C$ to denote a various constant, and $C_{\eta}$ when $C$ depends on $\eta . \quad c(*, \ldots, *)$ denotes a constant depending on the quantities appearing in parentheses such that $c(*, \ldots, *)$ increases with the quantities. Especially, $c\left(u_{0}, *, \ldots, *\right)$ denotes a constant depending on $\int_{\boldsymbol{R}^{2}}\left(2+u_{0}\right) \log \left(2+u_{0}\right) d x$ and the other quantities appearing in parentheses such that $c\left(u_{0}, *, \ldots, *\right)$ increases with these quantities. The Lebesgue measure is written by $|A|$, where $A$ is a measurable set in $\boldsymbol{R}^{n} . B_{r}(x)$ stands for the Euclidean open ball of radius $r$ centered at $x$ and $B_{r}=B_{r}(0)$. 
This paper is organized as follows. In Section 2 we give some function inequalities such as the Sobolev inequalities, the BMO estimate, the HardyLittlewood-Sobolev inequality and the Brezis-Merle inequality. In Section 3 we establish the a priori estimate of the modified entropy $\int_{R^{2}}(1+u) \log (1+u) d x$ for the nonnegative solution (see Theorem 3.1), which implies the global existence of the solution. Subsections 3.1 and 3.2 are devoted to the a priori estimates in the exterior region, Subsection 3.3 in the annulus and Subsection 3.4 in the interior region.

\section{Preliminary}

Before proving our main theorem, we prepare some function inequalities.

Lemma 2.1. (1) Let $1 \leq q<\infty$. There exists a positive constant $C$ depending only on $q$ such that for any $g \in L^{1}\left(\boldsymbol{R}^{2}\right)$ with $|\nabla g| \in L^{2}\left(\boldsymbol{R}^{2}\right)$,

$$
\|g\|_{q} \leq C\|g\|_{1}^{1-\lambda}\|\nabla g\|_{2}^{\lambda}, \quad \lambda=1-\frac{1}{q} .
$$

(2) There exists a positive constant $C$ such that for any $g \in L^{2}\left(\boldsymbol{R}^{2}\right)$ with $|\nabla g| \in L^{1}\left(\boldsymbol{R}^{2}\right)$,

$$
\|g\|_{2} \leq \frac{1}{\sqrt{2}}\|\nabla g\|_{1}
$$

For this lemma, see [24, 49] for example.

Lemma 2.2. For any $f \in W^{2,2}\left(\boldsymbol{R}^{2}\right)$ and nonnegative cut-off function $\phi \in C_{0}^{\infty}\left(\boldsymbol{R}^{2}\right)$, we have the following estimates:

(1)

$$
\begin{aligned}
\int_{\boldsymbol{R}^{2}}|f|^{2} \phi d x \leq & 2\left(\int_{\{|f|>1\} \cap \operatorname{supp}[\phi]}|f| d x\right)\left(\int_{|f|>1} \frac{|\nabla f|^{2}}{1+|f|} \phi d x\right) \\
& +\left(\int_{\boldsymbol{R}^{2}}|f|\left|\nabla \phi^{1 / 2}\right| d x\right)^{2}+4\left(\int_{\boldsymbol{R}^{2}}|f| \phi d x\right)
\end{aligned}
$$

(2)

$$
\begin{aligned}
\int_{\boldsymbol{R}^{2}}|f|^{2} \phi d x \leq & \varepsilon\left(\int_{\operatorname{supp}[\phi]}(1+|f|) \log (1+|f|) d x\right)\left(\int_{\boldsymbol{R}^{2}} \frac{|\nabla f|^{2}}{1+|f|} \phi d x\right) \\
& +4\left(\int_{\boldsymbol{R}^{2}}\left|f \nabla \phi^{1 / 2}\right| d x\right)^{2}+C(\varepsilon)\left(\int_{\boldsymbol{R}^{2}} f \phi d x\right)
\end{aligned}
$$

where $\varepsilon$ is any positive number and $C(\varepsilon)=O\left(e^{\varepsilon^{-1}}\right)$ as $\varepsilon \rightarrow 0$. 
(3)

$$
\begin{aligned}
\int_{\boldsymbol{R}^{2}}|f|^{3} \phi d x \leq & \varepsilon\left(\int_{\operatorname{supp}[\phi]}(1+|f|) \log (1+|f|) d x\right)\left(\int_{\boldsymbol{R}^{2}}|\nabla f|^{2} \phi d x\right) \\
& +C\left(\int_{\boldsymbol{R}^{2}}|f|^{3 / 2}\left|\nabla \phi^{1 / 2}\right| d x\right)^{2}+C(\varepsilon) \int_{\boldsymbol{R}^{2}}|f| \phi d x
\end{aligned}
$$

where $\varepsilon$ is any positive number and $C(\varepsilon)=O\left(e^{3 \varepsilon^{-1}}\right)$ as $\varepsilon \rightarrow 0$.

Proof. Without losing generality, we assume that $f$ is a nonnegative function. Now since $\phi(x) \geq 0$, setting $d \mu(x)=\phi(x) d x$ and letting $\mu_{f}(t)=$ $\mu(\{x ; f(x)>t\})$ for $f \geq 0$, we have

$$
\begin{aligned}
\int_{\boldsymbol{R}^{2}}|f(x)|^{2} d \mu & =2 \int_{\alpha}^{\infty} t \mu_{f}(t) d t+2 \int_{0}^{\alpha} t \mu_{f}(t) d t \\
& \leq 2 \int_{0}^{\infty}(t+\alpha) \mu_{f}(t+\alpha) d t+2 \int_{0}^{\alpha} t \mu_{f}(t) d t \\
& \leq 2 \int_{0}^{\infty} t \mu_{f-\alpha}(t) d t+2 \alpha \int_{0}^{\infty} \mu_{f-\alpha}(t) d t+2 \int_{0}^{\alpha} t \mu_{f}(t) d t \\
& \leq 2 \int_{f>\alpha}|f-\alpha|^{2} d \mu+2 \alpha \int_{f>\alpha}|f-\alpha| d \mu+2 \alpha \int_{0}^{\alpha} \mu_{f}(t) d t .
\end{aligned}
$$

By applying (2.2) as $g=(f-\alpha)_{+} \phi^{1 / 2}$, where $(f-\alpha)_{+}$is the positive part of $f-\alpha$, it follows that

$$
\begin{aligned}
\int_{\boldsymbol{R}^{2}}|f(x)|^{2} \phi d x \leq & 2 \int_{f>\alpha}|f-\alpha|^{2} \phi d x+4 \alpha \int_{\boldsymbol{R}^{2}}|f| \phi d x \\
\leq & \left(\int_{f>\alpha}|\nabla f| \phi^{1 / 2} d x\right)^{2} \\
& +\left(\int_{f>\alpha}|f|\left|\nabla \phi^{1 / 2}\right| d x\right)^{2}+4 \alpha \int_{\boldsymbol{R}^{2}}|f| \phi d x .
\end{aligned}
$$

To show (2.3), we take $\alpha=1$ in (2.6) and use Hölder's inequality to get

$$
\begin{aligned}
\left(\int_{f>1}|\nabla f| \phi^{1 / 2} d x\right)^{2} & \leq\left(\int_{\{f>1\} \cap \operatorname{supp}[\phi]} f d x\right)\left(\int_{f>1} \frac{1}{f}|\nabla f|^{2} \phi d x\right) \\
& \leq 2\left(\int_{\{f>1\} \cap \operatorname{supp}[\phi]} f d x\right)\left(\int_{f>1} \frac{|\nabla f|^{2}}{1+f} \phi d x\right) .
\end{aligned}
$$

Hence we have (2.3). 
Next, to show (2.4), by Hölder's inequality we calculate as follows.

$$
\begin{aligned}
& \left(\int_{f>\alpha}|\nabla f| \phi^{1 / 2} d x\right)^{2} \\
& \quad \leq\left(\int_{\{f>\alpha\} \cap \operatorname{supp}[\phi]}(1+f) d x\right)\left(\int_{f>\alpha} \frac{|\nabla f|^{2}}{1+f} \phi d x\right) \\
& \quad \leq \frac{1}{\log (1+\alpha)}\left(\int_{\{f>\alpha\} \cap \operatorname{supp}[\phi]}(1+f) \log (1+f) d x\right)\left(\int_{f>\alpha} \frac{|\nabla f|^{2}}{1+f} \phi d x\right) \\
& \quad \leq \frac{1}{\log (1+\alpha)}\left(\int_{\operatorname{supp}[\phi]}(1+f) \log (1+f) d x\right)\left(\int_{R^{2}} \frac{|\nabla f|^{2}}{1+f} \phi d x\right) .
\end{aligned}
$$

Putting $\varepsilon^{-1}=\log (1+\alpha)$, we conclude (2.4).

Using calculations similar to (2.5), we have

$$
\int_{\boldsymbol{R}^{2}}|f(x)|^{3} \phi(x) d x \leq \frac{5}{3} \int_{f>\alpha}|f-\alpha|^{3} \phi d x+18 \alpha^{2} \int_{f>\alpha}|f| \phi d x .
$$

Applying (2.2) as $g=(f-\alpha)_{+}^{3 / 2} \phi^{1 / 2}$ yields that

$$
\begin{aligned}
& \int_{f>\alpha}|f-\alpha|^{3} \phi d x \\
& \leq \frac{1}{2}\left(\int_{f>\alpha}\left|\nabla\left\{(f-\alpha)^{3 / 2} \phi^{1 / 2}\right\}\right| d x\right)^{2} \\
& \leq \frac{9}{4}\left(\int_{f>\alpha}|f-\alpha|^{1 / 2}|\nabla f| \phi^{1 / 2} d x\right)^{2}+\left(\int_{f>\alpha}|f-\alpha|^{3 / 2}\left|\nabla \phi^{1 / 2}\right| d x\right)^{2} \\
& \leq 3\left(\int_{\{f>\alpha\} \cap \operatorname{supp}[\phi]}|f-\alpha| d x\right)\left(\int_{f>\alpha}|\nabla f|^{2} \phi d x\right)+\left(\int_{f>\alpha}|f|^{3 / 2}\left|\nabla \phi^{1 / 2}\right| d x\right)^{2} \\
& \leq \frac{3}{\log (1+\alpha)}\left(\int_{\{f>\alpha\} \cap \operatorname{supp}[\phi]}(1+f) \log (1+f) d x\right)\left(\int_{f>\alpha}|\nabla f|^{2} \phi d x\right) \\
&+\left(\int_{f>\alpha}|f|^{3 / 2}\left|\nabla \phi^{1 / 2}\right| d x\right)^{2} .
\end{aligned}
$$

This implies the desired estimate by putting $\varepsilon=3 / \log (1+\alpha)$.

Lemma 2.3. Let $f$ be a nonnegative measurable function on a measurable set $\Omega$ in $\boldsymbol{R}^{n}$ satisfying $f \log (2+|x|) \in L^{1}(\Omega)$. The following conditions (i) and (ii) are equivalent. 
(i) $f \log f \in L^{1}(\Omega)$,

(ii) $(f+1) \log (f+1) \in L^{1}(\Omega)$.

More precisely,

$$
\begin{aligned}
& \int_{\Omega}(f+1) \log (f+1) d x \leq 2 \int_{\Omega} f|\log f| d x+(2 \log 2) \int_{\Omega} f d x \\
& \int_{\Omega} f|\log f| d x \leq \\
& \int_{\Omega}(f+1) \log (f+1) d x \\
& +2 \alpha \int_{\Omega} f \log (2+|x|) d x+\frac{1}{e} \int_{\Omega} \frac{1}{(2+|x|)^{\alpha}} d x
\end{aligned}
$$

where $n<\alpha<\infty$.

Proof. By the inequality

$$
(1+a) \log (1+a) \leq 2 a|\log a|+(2 \log 2) a, \quad a \geq 0,
$$

we have

$$
(1+f(x)) \log (1+f(x)) \leq 2 f(x)|\log f(x)|+(2 \log 2) f(x) .
$$

Integrating this on $\Omega$ implies (2.7).

We claim the following inequality: For $a \geq 0, b>0$,

$$
a|\log a| \leq(a+1) \log (a+1)+2 a|\log b|+e^{-1} b .
$$

In fact, $|(a / b) \log (a / b)| \leq e^{-1}$ for $a / b \leq 1$. Hence $a|\log a| \leq e^{-1} b+a|\log b|$. Next, for $a / b>1, \quad|\log (a / b)| \leq|\log ((a+1) / b)|$, from which it follows that $|\log a| \leq \log (a+1)+2|\log b|$. Hence $a|\log a| \leq(a+1) \log (a+1)+2 a|\log b|$. Thus we obtain the claim.

Put $a=f(x), b=(2+|x|)^{-\alpha} \quad(n<\alpha<\infty)$ in (2.9). Then $f(x)|\log f(x)| \leq(f(x)+1) \log (f(x)+1)+2 \alpha f(x) \log (2+|x|)+e^{-1}(2+|x|)^{-\alpha}$. Integrating this on $\Omega$ implies (2.8).

We next consider the Poisson equation

$$
\left\{\begin{array}{l}
-\Delta \psi=f \\
f \in L^{1}\left(\boldsymbol{R}^{2}\right)
\end{array} \text { in } \boldsymbol{R}^{2},\right.
$$

and define the solution of the Poisson equation by the Newtonian potential

$$
\psi=\frac{1}{2 \pi} \log |x|^{-1} * f \quad \text { in } \mathscr{S}^{*},
$$


where $\mathscr{S}^{*}$ is the space of tempered distributions on $\boldsymbol{R}^{2}$. Let $L \log L\left(\boldsymbol{R}^{n}\right)$ be the Orlicz class of functions defined by

$$
\int_{\boldsymbol{R}^{n}}(1+|f(x)|) \log (1+|f(x)|) d x<\infty,
$$

and $B M O\left(\boldsymbol{R}^{n}\right)$ the class of functions of the bounded mean oscillations defined by

$$
\|f\|_{B M O} \equiv \sup _{R>0, x} \frac{1}{\left|B_{R}\right|} \int_{B_{R}(x)}\left|f(y)-[f]_{B_{R}(x)}\right| d y<\infty,
$$

where $[f]_{B_{R}(x)}$ is the average of $f$ over the ball $B_{R}(x)$ of radius $R$ centered at $x$ and $\left|B_{R}\right|$ is the Lebesgue measure of $B_{R}$. For simplicity we denote $L \log L\left(\boldsymbol{R}^{2}\right)$ and $B M O\left(\boldsymbol{R}^{2}\right)$ by $L \log L$ and $B M O$, respectively. If $f$ satisfies $f \in L \log L$ then the solution $\psi$ becomes a locally bounded function and if $f \log (1+|x|) \in$ $L^{1}\left(\boldsymbol{R}^{2}\right)$ then the solution is locally integrable, and in both case, $\psi$ satisfies the Poisson equation in the sense of distribution. Note that in this case

$$
\nabla \psi(x)=-\frac{1}{2 \pi} \int_{\boldsymbol{R}^{2}} \frac{x-y}{|x-y|^{2}} f(y) d y \quad \text { a.e. in } \boldsymbol{R}^{2} .
$$

The following proposition is well known for the solution of two dimensional Poisson equation:

Proposition 2.4 (BMO estimate). Let $f \log (2+|x|) \in L^{1}\left(\boldsymbol{R}^{2}\right)$ and $\psi$ be a corresponding solution of the Poisson equation given by (2.10). Then we have that $\psi \in B M O$ and

$$
\|\psi\|_{B M O} \leq C\|f\|_{1},
$$

where $C$ is a constant independent of $f$.

Remark. This is well known and can be generalize such that $f$ is in a class of the Radon measure or the higher dimension cases $n \geq 3$ with $f \in L^{n / 2}\left(\boldsymbol{R}^{n}\right)$. The Brezis-Merle inequality for the elliptic case is a corollary of the above theorem and the well known John-Nirenberg inequality.

The following lemma is frequently used in the course of the proofs. For the proof of the lemma, see [63] for example.

Lemma 2.5. Let $\psi \in B M O\left(\boldsymbol{R}^{2}\right)$ and $\psi_{m}=\psi-[\psi]_{B_{R}}$, where $[\psi]_{B_{R}}$ is the average of $\psi$ over a ball $B_{R}$. Then, for any $1 \leq p<\infty$,

$$
\left(R^{-2} \int_{B_{R}}\left|\psi_{m}\right|^{p} d x\right)^{1 / p} \leq C\|\psi\|_{B M O}
$$

where $C$ is a positive constant depending only on $p$. 
In order to obtain a priori estimates for $\nabla \psi$, we need the HardyLittlewood-Sobolev inequality (see [63] for example).

Lemma 2.6 (The Hardy-Littlewood-Sobolev inequality). Let $0<\mu<n$ and $1<p, q<\infty$ with $1 / q=1 / p-\mu / n$. Then there exists a positive constant $C$ depending only on $p, q, n, \mu$ such that for any $f \in L^{p}\left(\boldsymbol{R}^{n}\right)$,

$$
\left\||x|^{-n+\mu} * f\right\|_{L^{q\left(\boldsymbol{R}^{n}\right)}} \leq C\|f\|_{L^{p}\left(\boldsymbol{R}^{n}\right)},
$$

where the symbol * stands for the convolution.

The following lemma about the Poisson equation in $\boldsymbol{R}^{2}$ is a consequence of the Brezis-Merle inequality under zero Dirichlet boundary conditions.

Lemma 2.7. Let $\Omega$ be a bounded domain in $\boldsymbol{R}^{2}$ with smooth boundary. For $g \in L^{2}(\Omega)$, let $v \in W^{2,2}(\Omega)$ be a solution of $-\Delta v=g$ in $\Omega$. If $\|g\|_{L^{1}(\Omega)}<4 \pi$, then

$$
\int_{\Omega} e^{|v(x)|} d x \leq \frac{4 \pi^{2}}{4 \pi-\|g\|_{L^{1}(\Omega)}} \mathrm{d}(\Omega)^{2} \exp \left\{\sup _{\partial \Omega}|v(x)|\right\},
$$

where $\mathrm{d}(\Omega)$ is the diameter of $\Omega$.

Proof. Let $w \in W^{2,2}(\Omega) \cap W_{0}^{1,2}(\Omega)$ be a solution of

$$
-\Delta w=g \quad \text { in } \Omega, \quad w=0 \quad \text { on } \partial \Omega .
$$

We recall the Brezis-Merle inequality (Theorem 1, [16]): For every $\delta \in(0,4 \pi)$,

$$
\int_{\Omega} \exp \left\{\frac{(4 \pi-\delta)|w(x)|}{\|g\|_{L^{1}(\Omega)}}\right\} d x \leq \frac{4 \pi^{2}}{\delta} \mathrm{d}(\Omega)^{2} .
$$

Taking $\delta=4 \pi-\|g\|_{L^{1}(\Omega)}(>0)$ implies

$$
\int_{\Omega} e^{|w(x)|} d x \leq \frac{4 \pi^{2}}{4 \pi-\|g\|_{L^{1}(\Omega)}} \mathrm{d}(\Omega)^{2} .
$$

Since $\Delta(v-w)=0$ in $\Omega$, the maximum principle implies

$$
|v(x)-w(x)| \leq \sup _{\partial \Omega}|v-w|=\sup _{\partial \Omega}|v|, \quad x \in \Omega .
$$

Hence

$$
\int_{\Omega} e^{|v(x)|} d x \leq \int_{\Omega} e^{|w(x)|} e^{|v(x)-w(x)|} d x \leq \frac{4 \pi^{2}}{4 \pi-\|g\|_{L^{1}(\Omega)}} \mathrm{d}(\Omega)^{2} \exp \left\{\sup _{\partial \Omega}|v(x)|\right\} .
$$




\section{A priori estimates for the Cauchy problem (1.1)}

In this section, we assume that for given nontrivial initial data $u_{0}$ satisfying

$$
u_{0} \geq 0, \quad u_{0} \in L^{1}\left(\boldsymbol{R}^{2}\right), \quad \int_{\boldsymbol{R}^{2}} u_{0} \log (1+|x|) d x<\infty,
$$

there exists a time local nonnegative solution $u$ for the Cauchy problem (1.1) over arbitrary time interval $[0, T)$ with $\psi$ defined by $(1.4)$ such that $(u, \psi)$ is smooth and have a proper integrability. We prove the following theorem, which implies (1.12) and hence, the global existence of the nonnegative solution $(u, \psi)$.

Theorem 3.1. Under the assumption

$$
\int_{R^{2}} u_{0} d x \leq 8 \pi
$$

it holds that for $0<t_{0}<T$,

$$
\sup _{t_{0} \leq t<T} \int_{\boldsymbol{R}^{2}}(1+u(t)) \log (1+u(t)) d x<\infty .
$$

As we mentioned in the introduction, the proof of the a priori estimate (3.2) requires a careful treatment on the behavior of the solution $\psi$. In order to do this, we split the derivation of the crucial a priori estimate into two parts, namely, exterior region and interior region. We first prove (3.2) for the exterior regions (see Proposition 3.2) and then for the interior regions (see Proposition 3.16), and hence conclude Theorem 3.1.

Since the nontrivial nonnegative solution $u$ is positive on $\boldsymbol{R}^{2}$ for $0<t<T$ and has smooth properties mentioned in Proposition 1.1 and Remark, taking $t=t_{0} \in(0, T)$ as the initial time, throughout this section we may assume, in addition to (3.1), that $u_{0}$ is positive on $\boldsymbol{R}^{2}$ and

$$
\left(1+u_{0}\right) \log \left(1+u_{0}\right) \in L^{1}\left(\boldsymbol{R}^{2}\right), \quad u_{0} \in W^{k, p}\left(\boldsymbol{R}^{2}\right)
$$

for all $k \geq 0$ and all $1<p \leq \infty$.

\subsection{Entropy estimates for exterior regions}

We begin with the estimate for large $|x|$. In this case, the a priori estimate can be obtained by the smallness of the solution $u$ for sufficiently large $|x|$. For the solution $u$, let

$$
H_{\text {ext }}(t ; R) \equiv \int_{|x| \geq R}((1+u(t)) \log (1+u(t))-u(t)) d x
$$


Proposition 3.2. There exists a sufficiently large number $R_{0}$ depending only on $T$ and $\left\|u_{0}\right\|_{1}$ such that

$$
\begin{aligned}
& \sup _{0 \leq t<T} H_{\text {ext }}\left(t ; R_{0}\right)+\int_{0}^{T} \int_{|x| \geq R_{0}} \frac{|\nabla u(t)|^{2}}{1+u(t)} d x d t \leq 2 H_{\text {ext }}\left(0 ; R_{0} / 2\right)+c\left(\left\|u_{0}\right\|_{1}\right) T, \\
& \int_{0}^{T} \int_{|x| \geq R_{0}} u^{2}(t) d x d t \leq C\left\|u_{0}\right\|_{1} H_{\text {ext }}\left(0 ; R_{0} / 2\right)+c\left(\left\|u_{0}\right\|_{1}\right) T
\end{aligned}
$$

where $c\left(\left\|u_{0}\right\|_{1}\right)$ is a positive constant depending on $\left\|u_{0}\right\|_{1}$ and $C>0$ a constant independent of $u_{0}$ and $T$.

Remark 3.3. The assumption $\int_{R^{2}} u_{0} d x \leq 8 \pi$ is not required to prove Proposition 3.2.

Before proving this proposition, we introduce the Littlewood-Paley partition of the unity.

Definition 3.1. Let $\phi(r)$ be a function in $C_{0}^{\infty}(0, \infty)$ such that

$$
\phi(r)= \begin{cases}0, & 0 \leq r \leq 1 / 2, r \geq 2, \\ \leq 1, \text { smooth, positive, } & 1 / 2<r<2\end{cases}
$$

Then a family of the cut off functions $\left\{\Phi_{n}(x)\right\}_{n=0}^{\infty}$ is called as the LittlewoodPaley partition of the unity if they satisfies

$$
\Phi_{n}(x)=\phi\left(2^{-n}|x|\right), \quad x \in \boldsymbol{R}^{2}, n=0,1,2, \ldots
$$

and

$$
\sum_{n=0}^{\infty} \Phi_{n}(x)= \begin{cases}1, & 1<|x| \\ \text { smooth, positive, }, & 1 / 2<|x| \leq 1 \\ 0, & |x|<1 / 2\end{cases}
$$

The Littlewood-Paley decomposition of the unity has the following properties: Observing that $\phi^{1 / 6}(r)$ has the same support as $\phi(r)$, we have

$$
\left|\phi^{\prime}(r)\right|=6 \phi^{5 / 6}(r)\left|\left(\phi^{1 / 6}\right)^{\prime}(r)\right| \leq C \phi(r)^{5 / 6}, \quad\left|\phi^{\prime \prime}(r)\right| \leq C \phi(r)^{2 / 3} \quad(r \geq 0) .
$$

Then it follows from the construction of $\left\{\Phi_{n}\right\}_{n}$ that

$$
\Phi_{n}(x)= \begin{cases}0, & |x| \leq 2^{n-1},|x| \geq 2^{n+1} \\ \leq 1, \text { smooth, positive, } & 2^{n-1}<|x|<2^{n+1}\end{cases}
$$

and 


$$
\begin{aligned}
& \left|\partial_{x} \Phi_{n}\right| \leq C 2^{-n} \Phi_{n}^{5 / 6}, \quad\left|\partial_{x} \Phi_{n}^{1 / 2}\right| \leq C 2^{-n} \Phi_{n}^{1 / 3}, \\
& \left|\partial_{x}^{2} \Phi_{n}\right| \leq C 2^{-2 n} \Phi_{n}^{2 / 3},
\end{aligned}
$$

where $C>0$ is a constant independent of $n$.

We also denote the annulus by

$$
A_{n}=\left\{x \in \boldsymbol{R}^{2} ; 2^{n-1} \leq|x| \leq 2^{n+1}\right\}
$$

so that $\operatorname{supp} \Phi_{n} \subset A_{n}$. If we set

$$
\tilde{\Phi}_{n}(x)=\Phi_{n-1}(x)+\Phi_{n}(x)+\Phi_{n+1}(x),
$$

then we have by its construction, that

$$
\tilde{\Phi}_{n}(x) \Phi_{n}(x)=\Phi_{n}(x)
$$

and

$$
\tilde{\Phi}_{n}(x)=1, \quad x \in A_{n} .
$$

Proof of Proposition 3.2. For fixed $n$, we let $\psi_{m}(t)=\psi(t)-[\psi(t)]_{B}$, where $[\psi(t)]_{B}=(1 /|B|) \int_{B} \psi(t) d x, B=B_{2^{n+2}}(0)$. The equation exchanging $\psi(t)$ into $\psi_{m}(t)$ also holds, that is, $-\Delta \psi_{m}=u$. We observe that by the equation of $u$,

$$
\partial_{t}\left\{((1+u) \log (1+u)-u) \Phi_{n}\right\}=\Delta u \log (1+u) \Phi_{n}-\left(\nabla \cdot\left(u \nabla \psi_{m}\right)\right) \log (1+u) \Phi_{n},
$$

and that

$$
\begin{aligned}
& \Delta u \log (1+u) \Phi_{n}= \nabla \cdot\left\{\nabla u \log (1+u) \Phi_{n}-((1+u) \log (1+u)-u) \nabla \Phi_{n}\right\} \\
&+((1+u) \log (1+u)-u) \Delta \Phi_{n}-\frac{|\nabla u|^{2}}{1+u} \Phi_{n} \\
&-\left(\nabla \cdot\left(u \nabla \psi_{m}\right)\right) \log (1+u) \Phi_{n}=-\nabla \cdot\left\{u \log (1+u) \nabla \psi_{m} \Phi_{n}\right\} \\
&+u \log (1+u) \nabla \psi_{m} \cdot \nabla \Phi_{n} \\
&+u \nabla \log (1+u) \cdot \nabla \psi_{m} \Phi_{n} .
\end{aligned}
$$

Hence

$$
\begin{aligned}
\partial_{t}\{((1+u) & \left.\log (1+u)-u) \Phi_{n}\right\}+\frac{|\nabla u|^{2}}{1+u} \Phi_{n} \\
= & \nabla \cdot\left\{\nabla u \log (1+u) \Phi_{n}-((1+u) \log (1+u)-u) \nabla \Phi_{n}\right. \\
& \left.\quad-u \log (1+u) \nabla \psi_{m} \Phi_{n}\right\} \\
& +((1+u) \log (1+u)-u) \Delta \Phi_{n}+u \log (1+u) \nabla \psi_{m} \cdot \nabla \Phi_{n} \\
& +u \nabla \log (1+u) \cdot \nabla \psi_{m} \Phi_{n}
\end{aligned}
$$


We rewrite the last two terms on the right hand side of (3.7) as follows.

$$
\begin{aligned}
& u \log (1+u) \nabla \psi_{m} \cdot \nabla \Phi_{n}+u \nabla \log (1+u) \cdot \nabla \psi_{m} \Phi_{n} \\
&= u \log (1+u) \nabla \psi_{m} \cdot \nabla \Phi_{n}+\nabla(u-\log (1+u)) \cdot \nabla \psi_{m} \Phi_{n} \\
&= \nabla \cdot\left\{(u-\log (1+u)) \nabla \psi_{m} \Phi_{n}\right\}+((1+u) \log (1+u)-u) \nabla \psi_{m} \cdot \nabla \Phi_{n} \\
&-(u-\log (1+u)) \Delta \psi_{m} \Phi_{n} \\
&= \nabla \cdot\left\{(u-\log (1+u)) \nabla \psi_{m} \Phi_{n}+((1+u) \log (1+u)-u) \psi_{m} \nabla \Phi_{n}\right\} \\
&-\log (1+u) \psi_{m} \nabla u \cdot \nabla \Phi_{n}-((1+u) \log (1+u)-u) \psi_{m} \Delta \Phi_{n} \\
&-(u-\log (1+u)) \Delta \psi_{m} \Phi_{n} .
\end{aligned}
$$

Put $-\Delta \psi_{m}=u$ to the last term on the right hand side of (3.8), substitute (3.8) into (3.7) and then integrate (3.7) on $\boldsymbol{R}^{2}$. We then have

$$
\begin{aligned}
\frac{d}{d t} \int_{\boldsymbol{R}^{2}} & ((1+u(t)) \log (1+u(t))-u(t)) \Phi_{n} d x+\int_{\boldsymbol{R}^{2}} \frac{|\nabla u(t)|^{2}}{1+u(t)} \Phi_{n} d x \\
= & \int_{\boldsymbol{R}^{2}}\left(u^{2}(t)-u(t) \log (1+u(t)) \Phi_{n} d x\right. \\
& +\int_{\boldsymbol{R}^{2}}((1+u(t)) \log (1+u(t))-u(t)) \Delta \Phi_{n} d x \\
& -\int_{\boldsymbol{R}^{2}} \log (1+u(t)) \psi_{m}(t) \nabla u(t) \cdot \nabla \Phi_{n} d x \\
& -\int_{\boldsymbol{R}^{2}}((1+u(t)) \log (1+u(t))-u(t)) \psi_{m}(t) \Delta \Phi_{n} d x \\
= & I+I I+I I I+I V .
\end{aligned}
$$

For the first term on the right hand side of (3.9), noting $0 \leq u^{2}-u \log (1+u)$, we apply (2.3) for the solution $u$ to have

$$
\begin{aligned}
|I| \leq & \int_{\boldsymbol{R}^{2}} u^{2}(t) \Phi_{n} d x \\
\leq & 2\left(\int_{A_{n}} u(t) d x\right)\left(\int_{\boldsymbol{R}^{2}} \frac{|\nabla u(t)|^{2}}{1+u(t)} \Phi_{n} d x\right)+\left(\int_{\boldsymbol{R}^{2}} u(t)\left|\nabla \Phi_{n}^{1 / 2}\right| d x\right)^{2} \\
& +4 \int_{\boldsymbol{R}^{2}} u(t) \Phi_{n} d x
\end{aligned}
$$

where

$$
A_{n}=\left\{x \in \boldsymbol{R}^{2} ; 2^{n-1} \leq|x| \leq 2^{n+1}\right\}
$$


Using (3.5) and the non-negativeness of the solution, we have

$$
\left(\int_{\boldsymbol{R}^{2}} u(t)\left|\nabla \Phi_{n}^{1 / 2}\right| d x\right)^{2} \leq C 2^{-2 n}\left(\int_{\boldsymbol{R}^{2}} u(t) d x\right)^{2}=C 2^{-2 n}\left\|u_{0}\right\|_{1}^{2} .
$$

Hence by (3.10) and (3.11),

$$
|I| \leq 2\left(\int_{A_{n}} u(t) d x\right)\left(\int_{\boldsymbol{R}^{2}} \frac{|\nabla u(t)|^{2}}{1+u(t)} \Phi_{n} d x\right)+C 2^{-2 n}\left\|u_{0}\right\|_{1}^{2}+4 \int_{\boldsymbol{R}^{2}} u(t) \Phi_{n} d x
$$

For the second term on the right hand side of (3.9), since

$$
0 \leq(1+u) \log (1+u)-u \leq C \begin{cases}u & (0 \leq u \leq 1) \\ u^{4 / 3} & (u \geq 1)\end{cases}
$$

by (3.5) and Hölder's inequality we have

$$
\begin{aligned}
|I I| & \leq C 2^{-2 n} \int_{0 \leq u \leq 1} u(t) \Phi_{n}^{2 / 3} d x+C 2^{-2 n} \int_{u \geq 1} u^{4 / 3}(t) \Phi_{n}^{2 / 3} d x \\
& \leq C 2^{-2 n}\left\|u_{0}\right\|_{1}+C 2^{-2 n}\left|A_{n}\right|^{1 / 3}\left(\int_{\boldsymbol{R}^{2}} u^{2}(t) \Phi_{n} d x\right)^{2 / 3} \\
& \leq \int_{\boldsymbol{R}^{2}} u^{2}(t) \Phi_{n} d x+C 2^{-2 n}\left\|u_{0}\right\|_{1}+C 2^{-4 n} .
\end{aligned}
$$

By the same estimate of $\int_{R^{2}} u^{2}(t) \Phi_{n} d x$ as in the estimate of $|I|$, it follows from (3.13) that

$$
\begin{aligned}
|I I| \leq & 2\left(\int_{A_{n}} u(t) d x\right)\left(\int_{\boldsymbol{R}^{2}} \frac{|\nabla u(t)|^{2}}{1+u(t)} \Phi_{n} d x\right) \\
& +C 2^{-2 n}\left(\left\|u_{0}\right\|_{1}+\left\|u_{0}\right\|_{1}^{2}\right)+4 \int_{\boldsymbol{R}^{2}} u(t) \Phi_{n} d x+C 2^{-4 n} .
\end{aligned}
$$

The third term on the right hand side of (3.9) is estimated as follows.

$$
\begin{aligned}
& |I I I| \leq C 2^{-n} \int_{A_{n}} \frac{|\nabla u(t)|}{\sqrt{1+u(t)}}(\sqrt{1+u(t)} \log (1+u(t)))\left|\psi_{m}(t)\right| \Phi_{n}^{5 / 6} d x \\
& \leq C 2^{-n}\left(\int_{R^{2}} \frac{|\nabla u(t)|^{2}}{1+u(t)} \Phi_{n} d x\right)^{1 / 2} \\
& \times\left(\int_{\boldsymbol{R}^{2}}(1+u(t))^{3 / 2}(\log (1+u(t)))^{3} \Phi_{n} d x\right)^{1 / 3}\left(\int_{A_{n}}\left|\psi_{m}(t)\right|^{6} d x\right)^{1 / 6} \\
& \leq \frac{\varepsilon}{2} \int_{\boldsymbol{R}^{2}} \frac{|\nabla u(t)|^{2}}{1+u(t)} \Phi_{n} d x+\frac{\varepsilon}{3} \int_{\boldsymbol{R}^{2}}(1+u(t))^{3 / 2}(\log (1+u(t)))^{3} \Phi_{n} d x \\
& +C \varepsilon^{-5} 2^{-6 n} \int_{A_{n}}\left|\psi_{m}(t)\right|^{6} d x,
\end{aligned}
$$


where $\varepsilon$ is an arbitrary number with $0<\varepsilon<1$. Noting that

$$
(1+u)^{3 / 2}(\log (1+u))^{3} \leq C \begin{cases}u & (0 \leq u \leq 1) \\ u^{2} & (u \geq 1)\end{cases}
$$

we have

$$
\begin{aligned}
\int_{\boldsymbol{R}^{2}}(1 & +u(t))^{3 / 2}(\log (1+u(t)))^{3} \Phi_{n} d x \\
\leq & C \int_{\boldsymbol{R}^{2}} u^{2}(t) \Phi_{n} d x+C \int_{\boldsymbol{R}^{2}} u(t) \Phi_{n} d x \\
\leq & C\left(\int_{A_{n}} u(t) d x\right)\left(\int_{\boldsymbol{R}^{2}} \frac{|\nabla u(t)|^{2}}{1+u(t)} \Phi_{n} d x\right)+C 2^{-2 n}\left(\int_{\boldsymbol{R}^{2}} u_{0} d x\right)^{2} \\
& +C(1+\varepsilon) \int_{\boldsymbol{R}^{2}} u(t) \Phi_{n} d x
\end{aligned}
$$

and by Lemma 2.5 ,

$$
2^{-6 n} \int_{A_{n}}\left|\psi_{m}(t)\right|^{6} d x \leq C 2^{-4 n}\|\psi(t)\|_{B M O}^{6} .
$$

Hence, by (3.15)-(3.17),

$$
\begin{aligned}
|I I I| \leq & \left(\frac{\varepsilon}{2}+C \int_{A_{n}} u(t) d x\right) \int_{\boldsymbol{R}^{2}} \frac{|\nabla u(t)|^{2}}{1+u(t)} \Phi_{n} d x \\
& +C 2^{-2 n}\left(\int_{\boldsymbol{R}^{2}} u_{0} d x\right)^{2}+C(1+\varepsilon) \int_{\boldsymbol{R}^{2}} u(t) \Phi_{n} d x \\
& +C \varepsilon^{-5} 2^{-4 n}\|\psi(t)\|_{B M O}^{6} .
\end{aligned}
$$

We proceed to estimate $I V$ as follows. By Lemma 2.5 and the analogous estimate in (3.16),

$$
\begin{aligned}
|I V| \leq & C 2^{-2 n} \int_{A_{n}}((1+u(t)) \log (1+u(t))-u(t))\left|\psi_{m}(t)\right| \Phi_{n}^{2 / 3} d x \\
\leq & C 2^{-2 n}\left(\int_{A_{n}}((1+u(t)) \log (1+u(t))-u(t))^{3 / 2} \Phi_{n} d x\right)^{2 / 3} \\
& \times\left(\int_{A_{n}}\left|\psi_{m}(t)\right|^{3} d x\right)^{1 / 3}
\end{aligned}
$$




$$
\begin{aligned}
\leq & C 2^{-2 n+2 n / 3}\|\psi(t)\|_{B M O} \\
& \times\left(\int_{\boldsymbol{R}^{2}}((1+u(t)) \log (1+u(t))-u(t))^{3 / 2} \Phi_{n} d x\right)^{2 / 3} \\
\leq & \int_{\boldsymbol{R}^{2}}((1+u(t)) \log (1+u(t))-u(t))^{3 / 2} \Phi_{n} d x+C 2^{-4 n}\|\psi(t)\|_{B M O}^{3} \\
\leq & \left(\int_{A_{n}} u(t) d x\right)\left(\int_{\boldsymbol{R}^{2}} \frac{|\nabla u(t)|^{2}}{1+u(t)} \Phi_{n} d x\right) \\
& +C \varepsilon 2^{-2 n}\left(\int_{\boldsymbol{R}^{2}} u_{0} d x\right)^{2}+C \varepsilon(1+\varepsilon) \int_{\boldsymbol{R}^{2}} u(t) \Phi_{n} d x \\
& +C 2^{-4 n}\|\psi(t)\|_{B M O}^{3} .
\end{aligned}
$$

Combining (3.12), (3.14), (3.18) and (3.19) yields that

$$
\begin{aligned}
&|I|+|I I|+|I I I|+|I V| \\
& \leq\left\{9 \varepsilon+C \int_{A_{n}} u(t) d x\right\} \int_{\boldsymbol{R}^{2}} \frac{|\nabla u(t)|^{2}}{1+u(t)} \Phi_{n} d x \\
&+C 2^{-2 n}\left(\left\|u_{0}\right\|_{1}+\left\|u_{0}\right\|_{1}^{2}\right)+C(1+\varepsilon)^{2} \int_{\boldsymbol{R}^{2}} u(t) \Phi_{n} d x \\
&+C \varepsilon^{-5} 2^{-4 n}\|\psi(t)\|_{B M O}^{6}+C 2^{-4 n}\|\psi(t)\|_{B M O}^{3}+C 2^{-4 n}
\end{aligned}
$$

where $\varepsilon$ is an arbitrary number with $0<\varepsilon<1$.

Now we estimate $\int_{A_{n}} u(t) d x$ by using $\tilde{\Phi}_{n}(x)=\Phi_{n-1}(x)+\Phi_{n}(x)+\Phi_{n+1}(x)$. Observing that

$$
\begin{aligned}
\frac{d}{d t} \int_{\boldsymbol{R}^{2}} u(t) \tilde{\Phi}_{n} d x= & \int_{\boldsymbol{R}^{2}} u(t) \Delta \tilde{\Phi}_{n} d x+\int_{\boldsymbol{R}^{2}} u(t) \nabla \psi(t) \cdot \nabla \tilde{\Phi}_{n} d x \\
= & \int_{\boldsymbol{R}^{2}} u(t) \Delta \tilde{\Phi}_{n} d x-\frac{1}{4 \pi} \int_{\boldsymbol{R}^{2}} \int_{\boldsymbol{R}^{2}} u(t, x) u(t, y) \frac{x-y}{|x-y|^{2}} \\
& \cdot\left\{\nabla \tilde{\boldsymbol{\Phi}}_{n}(x)-\nabla \tilde{\boldsymbol{\Phi}}_{n}(y)\right\} d y d x \\
\leq & \left\|\Delta \tilde{\Phi}_{n}\right\|_{\infty}\left\|u_{0}\right\|_{1}+\frac{1}{4 \pi}\left\|u_{0}\right\|_{1}^{2}\left\|\partial_{x}^{2} \tilde{\boldsymbol{\Phi}}_{n}\right\|_{\infty}
\end{aligned}
$$

we have

$$
\int_{\boldsymbol{R}^{2}} u(t) \tilde{\Phi}_{n} d x \leq \int_{\boldsymbol{R}^{2}} u_{0} \tilde{\Phi}_{n} d x+C 2^{-2 n} t\left\{\left\|u_{0}\right\|_{1}+\left\|u_{0}\right\|_{1}^{2}\right\}
$$


By this inequality and (3.6),

$$
\int_{A_{n}} u(t) d x \leq \int_{\boldsymbol{R}^{2}} u_{0} \tilde{\Phi}_{n} d x+C 2^{-2 n} t\left\{\left\|u_{0}\right\|_{1}+\left\|u_{0}\right\|_{1}^{2}\right\}
$$

Take $\varepsilon=1 / 36$ in (3.20). Given $T$, by (3.21) we can choose $N$ sufficiently large such that for all $n \geq N$,

$$
9 \varepsilon+C \sup _{0 \leq t<T} \int_{A_{n}} u(t) d x \leq \frac{1}{2} .
$$

Then, by (3.9) and (3.20),

$$
\begin{aligned}
& \frac{d}{d t}\left(\int_{\boldsymbol{R}^{2}}((1+u(t)) \log (1+u(t))-u(t)) \Phi_{n} d x\right)+\frac{1}{2} \int_{\boldsymbol{R}^{2}} \frac{|\nabla u(t)|^{2}}{1+u(t)} \Phi_{n} d x \\
& \quad \leq C(1+\varepsilon)^{2} \int_{\boldsymbol{R}^{2}} u(t) \Phi_{n} d x \\
& \quad+C 2^{-2 n}\left(\|\psi(t)\|_{B M O}^{6}+\|\psi(t)\|_{B M O}^{3}+\left\|u_{0}\right\|_{1}^{2}+\left\|u_{0}\right\|_{1}+1\right) .
\end{aligned}
$$

By using $\|\psi(t)\|_{B M O} \leq C\|u(t)\|_{1}=C\left\|u_{0}\right\|_{1}$ by virtue of Proposition 2.4, from (3.22) it follows that for every $n \geq N$ and for any $0<t<T$,

$$
\begin{aligned}
\int_{\boldsymbol{R}^{2}}((1 & +u(t)) \log (1+u(t))-u(t)) \Phi_{n} d x+\frac{1}{2} \int_{0}^{t} \int_{\boldsymbol{R}^{2}} \frac{|\nabla u|^{2}}{1+u} \Phi_{n} d x d s \\
\leq & \int_{\boldsymbol{R}^{2}}\left(\left(1+u_{0}\right) \log \left(1+u_{0}\right)-u_{0}\right) \Phi_{n} d x \\
& +C \int_{0}^{T} \int_{\boldsymbol{R}^{2}} u(t) \Phi_{n} d x d t \\
& +C 2^{-4 n}\left(\left\|u_{0}\right\|_{1}^{6}+\left\|u_{0}\right\|_{1}^{3}+\left\|u_{0}\right\|_{1}^{2}+\left\|u_{0}\right\|_{1}+1\right) T .
\end{aligned}
$$

Adding these for $n \geq N$, by $\sum_{n=N}^{\infty} \Phi_{n}(x)=1$ for $|x| \geq 2^{N}$, we have

$$
\begin{aligned}
& \int_{|x| \geq 2^{N}}((1+u(t)) \log (1+u(t))-u(t)) d x+\frac{1}{2} \int_{0}^{t} \int_{|x| \geq 2^{N}} \frac{|\nabla u|^{2}}{1+u} d x d t \\
& \leq \int_{|x| \geq 2^{N-1}}\left(\left(1+u_{0}\right) \log \left(1+u_{0}\right)-u_{0}\right) d x \\
& \quad+C\left(\left\|u_{0}\right\|_{1}+\left\|u_{0}\right\|_{1}^{2}+\left\|u_{0}\right\|_{1}^{3}+\left\|u_{0}\right\|_{1}^{6}+1\right) T .
\end{aligned}
$$


Putting $R_{0}=2^{N}$, (3.3) is deduced. Next, by calculations similar to those in (3.12),

$$
\begin{aligned}
\int_{\boldsymbol{R}^{2}} u^{2}(t) \Phi_{n} d x \leq & 2\left\|u_{0}\right\|_{1}\left(\int_{\boldsymbol{R}^{2}} \frac{|\nabla u(t)|^{2}}{1+u(t)} \Phi_{n} d x\right) \\
& +C 2^{-2 n}\left\|u_{0}\right\|_{1}^{2}+4 \int_{\boldsymbol{R}^{2}} u(t) \Phi_{n} d x
\end{aligned}
$$

Using (3.23) to estimate the first term on the right hand side of (3.24) and adding these for $n \geq N$, we conclude (3.4).

\subsection{A priori estimates for exterior regions}

Let $R_{0}$ be the positive number determined in Proposition 3.2. Then

$$
\begin{aligned}
& \sup _{0<t<T} \int_{|x| \geq R_{0}}(1+u(t)) \log (1+u(t)) d x \leq c\left(u_{0}, T\right), \\
& \int_{0}^{T} \int_{|x| \geq R_{0}} \frac{|\nabla u|^{2}}{1+u} d x d t \leq c\left(u_{0}, T\right), \\
& \int_{0}^{T} \int_{|x| \geq R_{0}} u^{2} d x d t \leq c\left(u_{0}, T\right),
\end{aligned}
$$

where $c\left(u_{0}, T\right)$ is a positive constant depending on $\left\|\left(2+u_{0}\right) \log \left(2+u_{0}\right)\right\|_{1}, T$ as mentioned in the introduction.

For $R>1$, let $\Phi_{R} \in C_{0}^{\infty}\left(\boldsymbol{R}^{2}\right)$ be such that

$$
\Phi_{R}(x)= \begin{cases}0, & |x| \leq R / 2 \\ \text { less than } 1, \text { positive, }, & R / 2<|x|<R \\ 1, & |x| \geq R\end{cases}
$$

and

$$
\left|\partial_{x} \Phi_{R}\right| \leq C R^{-1} \Phi_{R}^{5 / 6}, \quad\left|\partial_{x} \Phi_{R}^{1 / 2}\right| \leq C R^{-1} \Phi_{R}^{1 / 3}, \quad\left|\partial_{x}^{2} \Phi_{R}\right| \leq C R^{-2} \Phi_{R}^{2 / 3},
$$

where $C$ is a positive constant independent of $R$. We put

$$
\tilde{A}_{R}=\left\{x \in \boldsymbol{R}^{2} ; R / 2 \leq|x| \leq R\right\} .
$$

Lemma 3.4. For any $R \geq 2 R_{0}$,

$$
\sup _{0<t<T} \int_{|x| \geq R} u^{2}(t) d x+\frac{1}{2} \int_{0}^{T} \int_{|x| \geq R}|\nabla u|^{2} d x d t \leq \int_{R^{2}} u_{0} d x+c\left(u_{0}, T, R\right) .
$$


Proof. Let $\psi_{m}(t)=\psi(t)-[\psi(t)]_{B}$, where $[\psi(t)]_{B}=(1 /|B|) \int_{B} \psi(t) d x, B=$ $B_{2 R}(0)$. Multiply the equation of $u$ by $u \Phi_{R}$ and integrate on $\boldsymbol{R}^{2}$. Integrating by parts, we have

$$
\begin{aligned}
\frac{1}{2} \frac{d}{d t} \int_{\boldsymbol{R}^{2}} u^{2}(t) \Phi_{R} d x+\int_{\boldsymbol{R}^{2}}|\nabla u(t)|^{2} \Phi_{R} d x \\
=-\int_{\boldsymbol{R}^{2}} u \nabla u \cdot \nabla \Phi_{R} d x-\frac{1}{2} \int_{\boldsymbol{R}^{2}} \Delta \psi_{m} u^{2} \Phi_{R} d x+\frac{1}{2} \int_{\boldsymbol{R}^{2}} u^{2} \nabla \psi_{m} \cdot \nabla \Phi_{R} d x \\
=\frac{1}{2} \int_{\boldsymbol{R}^{2}} u^{2} \Delta \Phi_{R} d x+\frac{1}{2} \int_{\boldsymbol{R}^{2}} u^{3} \Phi_{R} d x-\frac{1}{2} \int_{\boldsymbol{R}^{2}} u^{2} \psi_{m} \Delta \Phi_{R} d x \\
\quad-\frac{1}{2} \int_{\boldsymbol{R}^{2}} \nabla u^{2} \cdot \nabla \Phi_{R} \psi_{m} d x \\
=\frac{1}{2} \int_{\boldsymbol{R}^{2}} u^{3} \Phi_{R} d x+\frac{1}{2} \int_{\boldsymbol{R}^{2}} u^{2}\left(1-\psi_{m}\right) \Delta \Phi_{R} d x-\int_{\boldsymbol{R}^{2}} u \nabla u \cdot \nabla \Phi_{R} \psi_{m} d x .
\end{aligned}
$$

Here we used $-\Delta \psi_{m}=u$. Now, by $\left|\Delta \Phi_{R}\right| \leq C R^{-2} \Phi_{R}^{2 / 3}$ and Lemma 2.5, we have

$$
\begin{aligned}
\frac{1}{2} \int_{\boldsymbol{R}^{2}} u^{2}\left(1-\psi_{m}\right) \Delta \Phi_{R} d x & \leq C R^{-2}\left(\int_{\tilde{A}_{R}}\left(1+\left|\psi_{m}\right|\right)^{3} d x\right)^{1 / 3}\left(\int_{\boldsymbol{R}^{2}} u^{3} \Phi_{R} d x\right)^{2 / 3} \\
& \leq \frac{1}{4} \int_{\boldsymbol{R}^{2}} u^{3} \Phi_{R} d x+C R^{-6} \int_{\tilde{A}_{R}}\left(1+\left|\psi_{m}\right|\right)^{3} d x \\
& \leq \frac{1}{4} \int_{\boldsymbol{R}^{2}} u^{3} \Phi_{R} d x+C R^{-4}\left(\|\psi(t)\|_{B M O}^{3}+1\right)
\end{aligned}
$$

and by $\left|\nabla \Phi_{R}\right| \leq C R^{-1} \Phi_{R}^{5 / 6}$,

$$
\begin{aligned}
-\int_{\boldsymbol{R}^{2}} u \nabla u \cdot \nabla \Phi_{R} \psi_{m} d x & \leq C R^{-1}\left\|\psi_{m}\right\|_{L^{6}\left(\tilde{A}_{R}\right)}\left(\int_{\boldsymbol{R}^{2}} u^{3} \Phi_{R} d x\right)^{1 / 3}\left(\int_{\boldsymbol{R}^{2}}|\nabla u|^{2} \Phi_{R} d x\right)^{1 / 2} \\
& \leq \frac{1}{3} \int_{\boldsymbol{R}^{2}} u^{3} \Phi_{R} d x+\frac{1}{2} \int_{\boldsymbol{R}^{2}}|\nabla u(t)|^{2} \Phi_{R} d x+C R^{-4}\|\psi(t)\|_{B M O}^{6} .
\end{aligned}
$$

Hence

$$
\begin{aligned}
& \frac{d}{d t} \int_{\boldsymbol{R}^{2}} u^{2} \Phi_{R} d x+\int_{\boldsymbol{R}^{2}}|\nabla u|^{2} \Phi_{R} d x \\
& \quad \leq 2 \int_{\boldsymbol{R}^{2}} u^{3} \Phi_{R} d x+C R^{-4}\left(\|\psi(t)\|_{B M O}^{6}+\|\psi(t)\|_{B M O}^{3}+1\right) .
\end{aligned}
$$

We apply (3) of Lemma 2.2 to the first term on the right hand side of (3.30) and get 


$$
\begin{aligned}
\frac{d}{d t} \int_{\boldsymbol{R}^{2}} & u^{2} \Phi_{R} d x+\left(1-\varepsilon \int_{\tilde{A}_{R}}(1+u) \log (1+u) d x\right) \int_{\boldsymbol{R}^{2}}|\nabla u|^{2} \Phi_{R} d x \\
\leq & C\left(\int_{\boldsymbol{R}^{2}} u^{3 / 2}\left|\nabla \Phi_{R}^{1 / 2}\right| d x\right)^{2}+C(\varepsilon) \int_{\boldsymbol{R}^{2}} u \Phi_{R} d x \\
& +C R^{-4}\left(\|\psi(t)\|_{B M O}^{6}+\|\psi(t)\|_{B M O}^{3}+1\right) .
\end{aligned}
$$

Let $R \geq 2 R_{0}$. We observe that

$$
\begin{aligned}
\left(\int_{R^{2}} u^{3 / 2}\left|\nabla \Phi_{R}^{1 / 2}\right| d x\right)^{2} & \leq C R^{-2}\left(\int_{|x| \geq R / 2} u^{3 / 2} d x\right)^{2} \\
& \leq C R^{-2}\left(\int_{R^{2}} u d x\right)\left(\int_{|x| \geq R / 2} u^{2} d x\right) \\
& \leq C R^{-2}\left\|u_{0}\right\|_{1} \int_{|x| \geq R / 2} u^{2} d x
\end{aligned}
$$

and that by (3.27),

$$
\begin{aligned}
\int_{0}^{T}\left(\int_{R^{2}} u^{3 / 2}\left|\nabla \Phi_{R}^{1 / 2}\right| d x\right)^{2} d t & \leq C R^{-2}\left\|u_{0}\right\|_{1} \int_{0}^{T} \int_{|x| \geq R / 2} u^{2} d x d t \\
& \leq c\left(u_{0}, T\right) R^{-2}
\end{aligned}
$$

Choosing $\varepsilon>0$ in (3.31) such that

$$
1-\varepsilon \sup _{0 \leq t<T} \int_{A_{R}}(1+u(t)) \log (1+u(t)) d x=\frac{1}{2}
$$

and integrating the resulting inequality from 0 to $t$ with respect to the time variable, by (3.32) we have

$$
\int_{\boldsymbol{R}^{2}} u^{2}(t) \Phi_{R} d x+\frac{1}{2} \int_{0}^{t} \int_{\boldsymbol{R}^{2}}|\nabla u|^{2} \Phi_{R} d x d \sigma \leq \int_{\boldsymbol{R}^{2}} u_{0}^{2} \Phi_{R} d x+c\left(u_{0}, T, R\right) .
$$

This implies (3.28).

The following lemma is a consequence of (1) of Lemma 2.2 and Lemma 3.4 .

Lemma 3.5. For any $R \geq 2^{2} R_{0}$,

$$
\int_{0}^{T} \int_{|x| \geq R} u^{4} d x d t \leq c\left(u_{0},\left\|u_{0}\right\|_{2}, T, R\right) .
$$


Proof. Apply (1) of Lemma 2.2 as $f=u^{2}$. Then

$$
\begin{aligned}
\int_{\boldsymbol{R}^{2}} u^{4} \Phi_{R} d x \leq & 2\left(\int_{|x| \geq R / 2} u^{2} d x\right)\left(\int_{u>1} \frac{\left|\nabla u^{2}\right|^{2}}{1+u^{2}} \Phi_{R} d x\right) \\
& +\left(\int_{\boldsymbol{R}^{2}} u^{2}\left|\nabla \Phi_{R}^{1 / 2}\right| d x\right)^{2}+4 \int_{\boldsymbol{R}^{2}} u^{2} \Phi_{R} d x
\end{aligned}
$$

We estimate the first two terms on the right hand side of (3.34) as follows.

$$
\begin{aligned}
& \left(\int_{|x| \geq R / 2} u^{2} d x\right)\left(\int_{u>1} \frac{\left|\nabla u^{2}\right|^{2}}{1+u^{2}} \Phi_{R} d x\right) \\
& \leq 4\left(\int_{|x| \geq R / 2} u^{2}(t) d x\right)\left(\int_{|x| \geq R / 2}|\nabla u|^{2} \Phi_{R} d x\right), \\
& \left(\int_{R^{2}} u^{2}\left|\nabla \Phi_{R}^{1 / 2}\right| d x\right)^{2} \leq C R^{-2}\left(\int_{|x| \geq R / 2} u^{2} d x\right)^{2} .
\end{aligned}
$$

Hence, by Lemma 3.4, we deduce (3.33).

Lemma 3.6. For any $R \geq 2^{3} R_{0}$,

$$
\begin{gathered}
\sup _{0<t<T} \int_{|x| \geq R} u^{3}(t) d x+\int_{0}^{T} \int_{|x| \geq R}\left|\nabla u^{3 / 2}\right|^{2} \Phi_{R} d x d t \\
\leq \int_{\boldsymbol{R}^{2}} u_{0}^{3} d x+c\left(u_{0},\left\|u_{0}\right\|_{2}, T, R\right) .
\end{gathered}
$$

Proof. Multiply the equation of $u$ by $u^{2} \Phi_{R}$ and integrate on $\boldsymbol{R}^{2}$. Then, by integration by parts, we have

$$
\begin{aligned}
& \frac{1}{3} \frac{d}{d t} \int_{\boldsymbol{R}^{2}} u^{3} \Phi_{R} d x+\frac{8}{9} \int_{\boldsymbol{R}^{2}}\left|\nabla u^{3 / 2}\right|^{2} \Phi_{R} d x \\
& \quad=\frac{1}{3} \int_{\boldsymbol{R}^{2}} u^{3} \Delta \Phi_{R} d x-\int_{\boldsymbol{R}^{2}} u^{2} \nabla \cdot\left(u \nabla \psi_{m}\right) \Phi_{R} d x .
\end{aligned}
$$

We rewrite the second term on the right hand side of the equality above as follows.

$$
\begin{aligned}
-\int_{\boldsymbol{R}^{2}} u^{2} \nabla \cdot\left(u \nabla \psi_{m}\right) \Phi_{R} d x & =-\frac{2}{3} \int_{\boldsymbol{R}^{2}} u^{3} \Delta \psi_{m} \Phi_{R} d x+\frac{1}{3} \int_{\boldsymbol{R}^{2}} u^{3} \nabla \psi_{m} \cdot \nabla \Phi_{R} d x \\
& =\frac{2}{3} \int_{\boldsymbol{R}^{2}} u^{4} \Phi_{R} d x+\frac{1}{3} \int_{\boldsymbol{R}^{2}} u^{3} \nabla \psi_{m} \cdot \nabla \Phi_{R} d x
\end{aligned}
$$


Hence

$$
\begin{aligned}
& \frac{d}{d t} \int_{\boldsymbol{R}^{2}} u^{3} \Phi_{R} d x+\frac{8}{3} \int_{\boldsymbol{R}^{2}}\left|\nabla u^{3 / 2}\right|^{2} \Phi_{R} d x \\
& \quad=\int_{\boldsymbol{R}^{2}} u^{3} \Delta \Phi_{R} d x+\int_{\boldsymbol{R}^{2}} u^{3} \nabla \psi_{m} \cdot \nabla \Phi_{R} d x+2 \int_{\boldsymbol{R}^{2}} u^{4} \Phi_{R} d x \\
& \quad \leq C R^{-2} \int_{|x| \geq R / 2} u^{3} d x+\int_{\boldsymbol{R}^{2}} u^{3} \nabla \psi_{m} \cdot \nabla \Phi_{R} d x+2 \int_{|x| \geq R / 2} u^{4} d x
\end{aligned}
$$

The second term on the right hand side of (3.36) is written as

$$
\begin{aligned}
\int_{\boldsymbol{R}^{2}} u^{3} \nabla \psi_{m} \cdot \nabla \Phi_{R} d x & =-2 \int_{\boldsymbol{R}^{2}} u^{3 / 2}\left(\nabla u^{3 / 2} \cdot \nabla \Phi_{R}\right) \psi_{m} d x-\int_{\boldsymbol{R}^{2}} u^{3} \psi_{m} \Delta \Phi_{R} d x \\
& =I+I I .
\end{aligned}
$$

By Hölder's inequality and Lemma 2.5, I is estimated as follows.

$$
\begin{aligned}
I & \leq C R^{-1}\left\|\psi_{m}(t)\right\|_{L^{8}\left(\tilde{A}_{R}\right)}\left(\int_{|x| \geq R / 2} u^{4} d x\right)^{3 / 8}\left(\int_{\boldsymbol{R}^{2}}\left|\nabla u^{3 / 2}\right|^{2} \Phi_{R} d x\right)^{1 / 2} \\
& \leq \frac{1}{2} \int_{\boldsymbol{R}^{2}}\left|\nabla u^{3 / 2}\right|^{2} \Phi_{R} d x+\frac{3}{8} \int_{|x| \geq R / 2} u^{4} d x+C R^{-8}\left\|\psi_{m}(t)\right\|_{L^{8}\left(\tilde{A}_{R}\right)}^{8} \\
& \leq \frac{1}{2} \int_{\boldsymbol{R}^{2}}\left|\nabla u^{3 / 2}\right|^{2} \Phi_{R} d x+\frac{3}{8} \int_{|x| \geq R / 2} u^{4} d x+C R^{-6}\|\psi(t)\|_{B M O}^{8} .
\end{aligned}
$$

Similarly,

$$
\begin{aligned}
I I & \leq C R^{-2}\left\|\psi_{m}(t)\right\|_{L^{4}\left(\tilde{A}_{R}\right)}\left(\int_{|x| \geq R / 2} u^{4} d x\right)^{3 / 4} \\
& \leq \frac{1}{4} \int_{|x| \geq R / 2} u^{4} d x+C R^{-6}\|\psi(t)\|_{B M O}^{4} .
\end{aligned}
$$

From (3.36) and the estimates on $I$ and $I I$, we have

$$
\begin{aligned}
\frac{d}{d t} \int_{\boldsymbol{R}^{2}} & u^{3} \Phi_{R} d x+\int_{\boldsymbol{R}^{2}}\left|\nabla u^{3 / 2}\right|^{2} \Phi_{R} d x \\
\leq & C R^{-2} \int_{|x| \geq R / 2} u^{3} d x+C \int_{|x| \geq R / 2} u^{4} d x \\
& +C R^{-6}\left(\|\psi(t)\|_{B M O}^{8}+\|\psi(t)\|_{B M O}^{4}\right) \\
= & F(t) .
\end{aligned}
$$


Since $\|\psi(t)\|_{B M O} \leq C\|u(t)\|_{1}=C\left\|u_{0}\right\|_{1}$ and

$$
\int_{|x| \geq R / 2} u^{3} d x \leq \int_{R^{2}} u d x+\int_{|x| \geq R / 2} u^{4} d x
$$

by Lemmas 3.4 and 3.5 we have

$$
\int_{0}^{T} F(t) d t \leq c\left(u_{0},\left\|u_{0}\right\|_{2}, T, R\right) .
$$

Therefore it follows from (3.37) that for $0<t<T$,

$$
\int_{\boldsymbol{R}^{2}} u^{3}(t) \Phi_{R} d x+\int_{0}^{t} \int_{\boldsymbol{R}^{2}}\left|\nabla u^{3 / 2}\right|^{2} \Phi_{R} d x d s \leq \int_{\boldsymbol{R}^{2}} u_{0}^{3} \Phi_{R} d x+c\left(u_{0},\left\|u_{0}\right\|_{2}, T, R\right),
$$

which implies (3.35).

\subsection{A priori estimates for annuli}

Let $R_{0}$ be the positive number determined in Proposition 3.2 and put

$$
A_{R}=\left\{x \in \boldsymbol{R}^{2} ; R / 2 \leq|x| \leq 2 R\right\} .
$$

Lemma 3.7. There exists $R_{1} \geq 2{ }^{5} R_{0}$ such that for any $R \geq R_{1}, \partial_{x} \psi$ and $\psi_{m}$ are bounded on $(0, T) \times A_{R}$, where $\psi_{m}(t)=\psi(t)-[\psi(t)]_{B}, \quad[\psi(t)]_{B}=$ $(1 /|B|) \int_{B} \psi(t) d x, B=B_{2 R}(0)$.

Proof. By the expression of $\psi$, we have

$$
2 \pi\left|\partial_{x} \psi(t, x)\right| \leq\left(\int_{|y|<R / 3}+\int_{R / 3<|y|<3 R}+\int_{|y|>3 R}\right) \frac{u(t, y)}{|x-y|} d y .
$$

Let $x \in A_{R}$, that is, $R / 2 \leq|x| \leq 2 R$. Then, $|x-y| \geq R / 6$ for $|y|<R / 3$ and $|x-y| \geq R$ for $|y|>3 R$, and hence

$$
\begin{aligned}
\int_{|y|<R / 3} \frac{u(t, y)}{|x-y|} d y+\int_{|y|>3 R} \frac{u(t, y)}{|x-y|} d y & \leq \frac{6}{R} \int_{|y|<R / 3} u(t, y) d y+\frac{1}{R} \int_{|y|>3 R} u(t, y) d y \\
& \leq \frac{7}{R} \int_{R^{2}} u(t, y) d y=\frac{7}{R}\left\|u_{0}\right\|_{1} .
\end{aligned}
$$

By Hölder's inequality,

$$
\begin{aligned}
\int_{R / 3<|y|<3 R} \frac{u(t, y)}{|x-y|} d y & \leq\left(\int_{R / 3<|y|<3 R}|x-y|^{-3 / 2} d y\right)^{2 / 3}\left(\int_{R / 3<|y|<3 R} u^{3}(t, y) d y\right)^{1 / 3} \\
& \leq C R^{1 / 3}\left(\int_{R / 3<|y|<3 R} u^{3}(t, y) d y\right)^{1 / 3} .
\end{aligned}
$$


Hence

$$
\left|\partial_{x} \psi(t, x)\right| \leq \frac{C}{R}\left\|u_{0}\right\|_{1}+C R^{1 / 3}\left(\int_{R / 3<|y|<3 R} u^{3}(t, y) d y\right)^{1 / 3},
$$

from which with Lemma 3.6 it follows that $\partial_{x} \psi$ is bounded on $(0, T) \times A_{R}$ for any $R \geq 2^{5} R_{0}$.

By Lemma 2.5 and Proposition 2.4, for $1<p<\infty$,

$$
\left\|\psi_{m}(t)\right\|_{L^{p}\left(A_{R}\right)} \leq C(2 R)^{-2 p}\|\psi(t)\|_{B M O} \leq C(2 R)^{-2 p}\|u(t)\|_{1}=C(2 R)^{-2 p}\left\|u_{0}\right\|_{1},
$$

and hence, by the boundedness of $\partial_{x} \psi$ on $(0, T) \times A_{R}$ for any $R \geq 2^{5} R_{0}, \psi_{m}(t)$ is in $W^{1, p}\left(A_{R}\right)$ for $2<p<\infty$ and for each $0<t<T$. Then the boundedness of $\psi_{m}$ on $(0, T) \times A_{R}$ follows from the Sobolev inequalities.

For $R \geq 1$, let $\Phi_{R} \in C_{0}^{\infty}\left(\boldsymbol{R}^{2}\right)$ be such that

$$
\Phi_{R}(x)= \begin{cases}0, & |x| \leq R / 3,|x| \geq 3 R \\ 1, & R / 2 \leq|x| \leq 2 R \\ \text { less than } 1, \text { positive, }, & R / 3<|x|<R / 2,2 R<|x|<3 R\end{cases}
$$

and

$$
\left|\partial_{x} \Phi_{R}\right| \leq C R^{-1} \Phi_{R}^{5 / 6}, \quad\left|\partial_{x} \Phi_{R}^{1 / 2}\right| \leq C R^{-1} \Phi_{R}^{1 / 3}, \quad\left|\partial_{x}^{2} \Phi_{R}\right| \leq C R^{-2} \Phi_{R}^{2 / 3},
$$

where $C$ is a positive constant independent of $R$. We put $w(t, x)=u(t, x) \Phi_{R}(x)$, which satisfies $w \in C\left([0, T) ; L^{2}\left(\boldsymbol{R}^{2}\right)\right) \cap L^{\infty}\left(0, T ; L^{2}\left(\boldsymbol{R}^{2}\right)\right)$ and

$$
\partial_{t} w-\Delta w=f, \quad 0<t<T, x \in \boldsymbol{R}^{2},
$$

where

$$
f=-\left(2 \nabla \Phi_{R}+\Phi_{R} \nabla \psi\right) \cdot \nabla u-\left(\Delta \Phi_{R}\right) u+\Phi_{R} u^{2} .
$$

Lemma 3.8. There exists $R_{2} \geq 3 R_{1}$ such that for any $R \geq R_{2}$, $u$ is bounded on $(0, T) \times A_{R}$.

Proof. By Lemmas in Subsection 3.2 and Lemma 3.7, for $R \geq 3 R_{1}, f$ defined by (3.39) is in $L^{2}\left(0, T ; L^{2}\left(\boldsymbol{R}^{2}\right)\right)$, and then by regularity theorems for parabolic equations,

$$
w \in L^{2}\left(0, T ; H^{2}\left(\boldsymbol{R}^{2}\right)\right), \quad \partial_{t} w \in L^{2}\left(0, T ; L^{2}\left(\boldsymbol{R}^{2}\right)\right) .
$$

By the Sobolev inequalities,

$$
w \in L^{\infty}\left(0, T ; L^{2}\left(\boldsymbol{R}^{2}\right)\right) \cap L^{2}\left(0, T ; W^{1, p}\left(\boldsymbol{R}^{2}\right)\right) \quad \text { for all } 1<p<\infty .
$$


Hence for $R \geq 3 R_{1}$, we have

$$
f \in L^{2}\left(0, T ; L^{p}\left(\boldsymbol{R}^{2}\right)\right) \quad \text { for all } 1<p<\infty .
$$

Since $w$ satisfies (3.38), $w$ is represented as

$$
w(t)=e^{t \Delta} w_{0}+\int_{0}^{t} e^{(t-s) \Delta} f(s) d s, \quad 0<t<T,
$$

where $w_{0}=u_{0} \Phi_{R}$. By $L^{\infty}-L^{q}$ estimates for $e^{t \Delta}$, for $2<q<\infty$ we have

$$
\begin{aligned}
\|w(t)\|_{\infty} & \leq\left\|w_{0}\right\|_{\infty}+C \int_{0}^{t}(t-s)^{-1 / q}\|f(s)\|_{q} d s \\
& \leq\left\|w_{0}\right\|_{\infty}+C\left(\int_{0}^{t}(t-s)^{-2 / q} d s\right)^{1 / 2}\left(\int_{0}^{t}\|f(s)\|_{q}^{2} d s\right)^{1 / 2} \\
& \leq\left\|w_{0}\right\|_{\infty}+C \frac{q}{q-2} t^{1 / 2-1 / q}\left(\int_{0}^{t}\|f(s)\|_{q}^{2} d s\right)^{1 / 2}
\end{aligned}
$$

This implies the boundedness of $u$ on $A_{R}$ from the definition of $w$.

Lemma 3.9. There exists $R_{3} \geq 3^{3} R_{2}$ such that for any $R \geq R_{3}, \partial_{x} u$ is bounded on $(0, T) \times A_{R}$.

Proof. We begin with proving the following: For any $R \geq 3 R_{1}$,

$$
\sup _{0<t<T}\left\|\partial_{x} u(t)\right\|_{L^{2}\left(A_{R}\right)}<\infty
$$

To prove this, by integration by parts we have

$$
\frac{1}{2} \frac{d}{d t} \int_{\boldsymbol{R}^{2}}|\nabla u|^{2} \Phi_{R} d x=-\int_{\boldsymbol{R}^{2}} \partial_{t} u \Delta u \Phi_{R} d x-\int_{\boldsymbol{R}^{2}} \partial_{t} u \nabla u \cdot \nabla \Phi_{R} d x .
$$

We put $\psi_{m}(t)=\psi(t)-[\psi(t)]_{B}, B=B_{2 R}(0)$ and

$$
\tilde{A}_{R}=\left\{x \in \boldsymbol{R}^{2} ; R / 3 \leq|x| \leq 3 R\right\} .
$$

By $\partial_{t} u=\Delta u-\nabla u \cdot \nabla \psi_{m}+u^{2}$ and Hölder's inequality, we have

$$
\begin{aligned}
-\int_{\boldsymbol{R}^{2}} \partial_{t} u \Delta u \Phi_{R} d x= & -\int_{\boldsymbol{R}^{2}}|\Delta u|^{2} \Phi_{R} d x+\int_{\boldsymbol{R}^{2}} \nabla u \cdot \nabla \psi_{m} \Delta u \Phi_{R} d x-\int_{\boldsymbol{R}^{2}} u^{2} \Delta u \Phi_{R} d x \\
\leq & -\frac{3}{4} \int_{\boldsymbol{R}^{2}}|\Delta u|^{2} \Phi_{R} d x+C\left\|\nabla \psi_{m}(t)\right\|_{L^{\infty}\left(\tilde{A}_{R}\right)}^{2} \int_{\tilde{A}_{R}}|\nabla u|^{2} d x \\
& +C \int_{\tilde{A}_{R}} u^{4} d x .
\end{aligned}
$$


Similarly,

$$
\begin{aligned}
-\int_{R^{2}} \partial_{t} u \nabla u \cdot \nabla \Phi_{R} d x= & -\int_{\boldsymbol{R}^{2}} \Delta u \nabla u \cdot \nabla \Phi_{R} d x+\int_{\boldsymbol{R}^{2}} \nabla u \cdot \nabla \psi_{m} \nabla u \cdot \nabla \Phi_{R} d x \\
& -\int_{\boldsymbol{R}^{2}} u^{2} \nabla u \cdot \nabla \Phi_{R} d x \\
\leq & C R^{-1} \int_{\boldsymbol{R}^{2}}|\Delta u||\nabla u| \Phi_{R}^{5 / 6} d x \\
& +C R^{-1}\left\|\nabla \psi_{m}(t)\right\|_{L^{\infty}\left(\tilde{A}_{R}\right)} \int_{\tilde{A}_{R}}|\nabla u|^{2} d x+C R^{-1} \int_{\tilde{A}_{R}} u^{2}|\nabla u| d x \\
\leq & \frac{1}{4} \int_{R^{2}}|\Delta u|^{2} \Phi_{R} d x+C\left(\left\|\nabla \psi_{m}(t)\right\|_{L^{\infty}\left(\tilde{A}_{R}\right)}+1\right) \int_{\tilde{A}_{R}}|\nabla u|^{2} d x \\
& +C \int_{\tilde{A}_{R}} u^{4} d x .
\end{aligned}
$$

Hence

$$
\begin{aligned}
& \frac{d}{d t} \int_{\boldsymbol{R}^{2}}|\nabla u|^{2} \Phi_{R} d x+\int_{\boldsymbol{R}^{2}}|\Delta u|^{2} \Phi_{R} d x \\
& \quad \leq C\left(\left\|\nabla \psi_{m}(t)\right\|_{L^{\infty}\left(\tilde{A}_{R}\right)}^{2}+\left\|\nabla \psi_{m}(t)\right\|_{L^{\infty}\left(\tilde{A}_{R}\right)}+1\right) \int_{\tilde{A}_{R}}|\nabla u|^{2} d x+C \int_{\tilde{A}_{R}} u^{4} d x \\
& \quad=F(t) .
\end{aligned}
$$

Then (3.43) follows from $\int_{0}^{T} F(t) d t<\infty$ for any $R \geq 3 R_{1}$ by virtue of Lemmas $3.4,3.7$ and 3.8 .

Next, by (3.43) we see that for any $R \geq 3^{2} R_{2}$,

$$
\sup _{0<t<T}\|f(t)\|_{2}<\infty .
$$

We observe the following expression

$$
\partial_{x} w(t)=e^{t \Delta} \partial_{x} w_{0}+\int_{0}^{t} \partial_{x} e^{(t-s) \Delta} f(s) d s, \quad 0<t<T .
$$

Let $2 \leq p<\infty$. Then

$$
\begin{aligned}
\left\|\partial_{x} w(t)\right\|_{p} & \leq\left\|\partial_{x} w_{0}\right\|_{p}+C \int_{0}^{t}(t-s)^{-1+1 / p}\|f(s)\|_{2} d s \\
& \leq\left\|\partial_{x} w_{0}\right\|_{p}+C p t^{1 / p} \sup _{0<s<T}\|f(s)\|_{2} .
\end{aligned}
$$


Hence we obtain $\sup _{0<t<T}\left\|\partial_{x} w(t)\right\|_{p}<\infty$, and hence

$$
\sup _{0<t<T}\left\|\partial_{x} u(t)\right\|_{L^{p}\left(A_{R}\right)}<\infty .
$$

By this estimate, for any $R \geq 3^{3} R_{2}$, we also have

$$
\sup _{0<t<T}\|f(t)\|_{p}<\infty \quad \text { for } 2 \leq p<\infty .
$$

Similarly, the $L^{\infty}$-norm of the integral term on the right hand side of (3.44) is estimated by

$$
\int_{0}^{t}(t-s)^{-1 / q-1 / 2}\|f(s)\|_{q} d s \leq \frac{1}{1 / 2-1 / q} t^{1 / 2-1 / q} \sup _{0<t<T}\|f(t)\|_{q},
$$

where $2<q<\infty$. Hence we get $\sup _{0<t<T}\left\|\partial_{x} w(t)\right\|_{\infty}<\infty$, which implies the boundedness of $\partial_{x} u$ on $(0, T) \times A_{R}$ for any $R \geq 3^{3} R_{2}$.

Lemma 3.10. There exists $R_{4} \geq 3 R_{3}$ such that for any $R \geq R_{4}, \partial_{x}^{2} \psi$ is bounded on $(0, T) \times A_{R}$.

Proof. Let $R \geq 3 R_{3}$. With $\Phi_{R}$ we write $\partial_{x_{j}} \psi$ as follows.

$$
\begin{aligned}
2 \pi \partial_{x_{j}} \psi(t, x) & =-\int_{\boldsymbol{R}^{2}} \frac{x_{j}-y_{j}}{|x-y|^{2}} u(t, y) d y \\
& =-\int_{\boldsymbol{R}^{2}} \frac{x_{j}-y_{j}}{|x-y|^{2}} u(t, y)\left(1-\Phi_{R}(y)\right) d y-\int_{\boldsymbol{R}^{2}} \frac{x_{j}-y_{j}}{|x-y|^{2}} u(t, y) \Phi_{R}(y) d y \\
& =I(t, x)+I I(t, x) .
\end{aligned}
$$

Let $x \in A_{R}$, that is, $R / 2 \leq|x| \leq 2 R$. Observing

$$
\partial_{x_{k}} I(t, x)=-\int_{\boldsymbol{R}^{2}}\left(\frac{\delta_{j k}}{|x-y|^{2}}-2 \frac{\left(x_{j}-y_{j}\right)\left(x_{k}-y_{k}\right)}{|x-y|^{4}}\right) u(t, y)\left(1-\Phi_{R}(y)\right) d y
$$

we have

$$
\begin{aligned}
\left|\partial_{x_{k}} I(t, x)\right| & \leq\left(\int_{|y|<R / 3}+\int_{|y|>3 R}\right) \frac{3}{|x-y|^{2}} u(t, y) d y \\
& \leq C R^{-2} \int_{\boldsymbol{R}^{2}} u(t, y) d y=C R^{-2}\left\|u_{0}\right\|_{1} .
\end{aligned}
$$

By the expression

$$
\partial_{x_{k}} I I(t, x)=-\int_{\boldsymbol{R}^{2}} \frac{x_{j}-y_{j}}{|x-y|^{2}} \partial_{y_{k}}\left(u(t, y) \Phi_{R}(y)\right) d y,
$$


we estimate as follows.

$$
\begin{aligned}
\left|\partial_{x_{k}} I I(t, x)\right| & \leq \int_{R / 3<|y|<3 R} \frac{1}{|x-y|}\left|\partial_{y_{k}}\left(u(t, y) \Phi_{R}(y)\right)\right| d y \\
& \leq\left(\int_{|x|<3 R} \frac{1}{|x-y|} d y\right) \sup _{0<t<T}\left\|u(t), \partial_{x} u(t)\right\|_{L^{\infty}(R / 3<|x|<3 R)} .
\end{aligned}
$$

Hence we obtain the boundedness of $\partial_{x}^{2} \psi$ on $(0, T) \times A_{R}$ by Lemmas 3.8 and 3.9 .

Lemma 3.11. There exists $R_{5} \geq 3^{2} R_{4}$ such that for any $R \geq R_{5}, \partial_{x}^{2} u$ is bounded on $(0, T) \times A_{R}$.

Proof. By the preceding lemmas, for any $R \geq R_{4}$ the function $f$ defined by (3.39) is estimated as

$$
\sup _{0<t<T}\|f(t)\|_{\infty}<\infty
$$

Applying regularity theorems for parabolic equations to $w$ satisfying (3.38), we have

$$
w \in L^{p}\left(0, T ; W^{2, p}\left(\boldsymbol{R}^{2}\right)\right) \cap W^{1, p}\left(0, T ; L^{p}\left(\boldsymbol{R}^{2}\right)\right), \quad 1<p<\infty,
$$

and hence, for any $R \geq 3 R_{4}$,

$$
\int_{0}^{T}\left\|\partial_{x} f(t)\right\|_{p}^{p} d t<\infty, \quad 1<p<\infty
$$

We observe

$$
\partial_{x}^{2} w(t)=e^{t \Delta} \partial_{x}^{2} w_{0}-\int_{0}^{t} \partial_{x} e^{(t-s) \Delta} \partial_{x} f(s) d s
$$

Let $2<p<\infty$. We estimate $L^{p}$-norms for the integral term as follows.

$$
\begin{aligned}
\int_{0}^{t}\left\|\partial_{x} e^{(t-s) \Delta} \partial_{x} f(s)\right\|_{p} d s & \leq C \int_{0}^{t}(t-s)^{-1 / 2}\left\|\partial_{x} f(s)\right\|_{p} d s \\
& \leq C\left(\int_{0}^{t}(t-s)^{-p^{\prime} / 2} d s\right)^{1 / p^{\prime}}\left(\int_{0}^{t}\left\|\partial_{x} f(s)\right\|_{p}^{p} d s\right)^{1 / p},
\end{aligned}
$$

where $1<p^{\prime}<2,1 / p+1 / p^{\prime}=1$. Hence

$$
\sup _{0<t<T}\left\|\partial_{x}^{2} u(t)\right\|_{L^{p}\left(A_{R}\right)}<\infty \quad \text { for } 2<p<\infty .
$$


By this estimates, for any $R \geq 3^{2} R_{4}$

$$
\sup _{0<t<T}\left\|\partial_{x} f(t)\right\|_{p}<\infty, \quad 1<p<\infty .
$$

Similarly, by this estimate,

$$
\int_{0}^{t}\left\|\partial_{x} e^{(t-s) \Delta} \partial_{x} f(s)\right\|_{\infty} d s \leq C \int_{0}^{t}(t-s)^{-1 / p-1 / 2} d s \sup _{0<t<T}\left\|\partial_{x} f(t)\right\|_{p},
$$

where $2<p<\infty$. Hence we obtain $\sup _{0<t<T}\left\|\partial_{x}^{2} w(t)\right\|_{\infty}<\infty$, and hence the boundedness of $\partial_{x}^{2} u$ on $(0, T) \times A_{R}$.

Proposition 3.12. There exists $\tilde{R}_{0}>R_{0}$ such that for any $R \geq \tilde{R}_{0}$, the following hold:

(1) $\partial_{t}^{k} \partial_{x}^{\ell} u(0 \leq 2 k+\ell \leq 2)$ are bounded on $(0, T) \times A_{R}$,

(2) $\psi_{m}$ and $\partial_{x}^{\ell} \psi(1 \leq \ell \leq 2)$ are bounded on $(0, T) \times A_{R}$, where $\psi_{m}(t)=$ $\psi(t)-[\psi(t)]_{B}, B=B_{2 R}(0)$,

(3) there exist $x_{0}$ satisfying $R<\left|x_{0}\right|<2 R$, and $\varepsilon_{0}>0, \delta>0$ such that

$$
u(t, x) \geq \delta \quad \text { for }\left|x-x_{0}\right| \leq \varepsilon_{0}, 0 \leq t<T .
$$

Proof. The assertions (1) and (2) follow from the preceding lemmas.

To prove (3), we first claim that $\partial_{x}^{\ell} u(0 \leq \ell \leq 2)$ are uniformly Hölder continuous on $[T / 2, T) \times \tilde{A}_{R}$, where $\tilde{A}_{R}:=\left\{x \in \boldsymbol{R}^{2} ; 2 R / 3 \leq|x| \leq 8 R / 3\right\}$. In fact, since $u$ satisfies $\partial_{t} u-\Delta u=-\nabla \cdot(u \nabla \psi)$ and $u \nabla \psi$ is bounded on $(0, T) \times$ $\left\{x \in \boldsymbol{R}^{2} ; R / 3<|x|<3 R\right\}$, applying the Hölder estimates for parabolic equations (see Theorem 10.1 in [38] for example), we see that $u$ is uniformly Hölder continuous on $[T / 2, T) \times \tilde{A}_{R}$. Similarly, by $\partial_{t}\left(\partial_{x} u\right)-\Delta\left(\partial_{x} u\right)=-\nabla \cdot\left(\partial_{x}(u \nabla \psi)\right)$ and the boundedness of $\partial_{x}(u \nabla \psi)$ in $(0, T) \times\left\{x \in \boldsymbol{R}^{2} ; R / 3<|x|<3 R\right\}$, the uniformly Hölder continuity of $\partial_{x} u$ in $[T / 2, T) \times \tilde{A}_{R}$ is deduced. As for $\partial_{x_{k}} \partial_{x_{j}} u$, putting $v=\partial_{x_{k}} \partial_{x_{j}} u$, we observe that

$$
\partial_{t} v-\Delta v+\nabla \psi \cdot \nabla v-2 u v=\partial_{x_{k}} g_{1}+g_{2},
$$

where $g_{1}=-\nabla u \cdot \nabla \partial_{x_{j}} \psi, g_{2}=-\nabla \partial_{x_{j}} u \cdot \nabla \partial_{x_{j}} \psi+2 \partial_{x_{k}} u \partial_{x_{j}} u$. Hence, by the boundedness of $g_{1}$ and $g_{2}$ on $(0, T) \times\left\{x \in \boldsymbol{R}^{2} ; R / 3<|x|<3 R\right\}, \partial_{x_{k}} \partial_{x_{j}} u$ is uniformly Hölder continuous on $[T / 2, T) \times \tilde{A}_{R}$.

By the uniformly Hölder continuity of $\partial_{x}^{\ell} u(0 \leq \ell \leq 2)$ on $[T / 2, T) \times \tilde{A}_{R}$, the limits of $\partial_{x}^{\ell} u \quad(0 \leq \ell \leq 2)$ exist on $\tilde{A}_{R}$ as $t \rightarrow T-0$. Hence we may assume that $\partial_{x}^{\ell} u(0 \leq \ell \leq 2)$ are continuous on $(0, T] \times \tilde{A}_{R}$. By the positivity of $u$ on $(t, x) \in[T / 2, T) \times\left\{x \in \boldsymbol{R}^{2} ; R \leq|x| \leq 2 R\right\}, u(T, x) \geq 0$ for $R \leq|x| \leq 2 R$. 
We next claim that there exist $x_{0}$ satisfying $R<\left|x_{0}\right|<2 R$, and $\varepsilon_{0}>0$, $\delta>0$ such that

$$
u(T, x) \geq \delta \quad \text { for }\left|x-x_{0}\right| \leq \varepsilon_{0} .
$$

Assume that $u(T, x)=0$ for all $R<|x|<2 R$. Then $\partial_{x}^{\ell} u(T, x)=0$ for all $R<|x|<2 R$ and $\ell=1,2$. By $\partial_{t} u=\Delta u-\nabla \psi \cdot \nabla u+u^{2}$ and the boundedness of $\nabla \psi$ on $[T / 2, T) \times\left\{x \in R^{2} ; R<|x|<2 R\right\}$, we obtain that for every $R<|x|<$ $2 R$,

$$
\partial_{t} u(t, \tilde{x}) \rightarrow 0 \quad \text { as }(t, \tilde{x}) \rightarrow(T, x) .
$$

Hence we may assume that $\partial_{t} u$ is continuous on $(0, T] \times \tilde{A}_{R}$. Since $u(T, x)=0$ for all $R<|x|<2 R$, the maximum principle implies $u(t, x)=0$ for all $T / 2 \leq$ $t \leq T, R<|x|<2 R$. This contradicts the positivity of $u$ on $(t, x) \in[0, T) \times$ $\left\{x \in \boldsymbol{R}^{2} ; R<|x|<2 R\right\}$. Therefore the claim is concluded.

By the positivity of $u$ in $(t, x) \in[0, T) \times\left\{x \in \boldsymbol{R}^{2} ; R<|x|<2 R\right\}$ and (3.46), the assertion (3) is concluded.

\subsection{Entropy estimates for interior regions}

We finally give the interior a priori estimate. This is a crucial estimate for proving the global existence of the solution. Let us define $\Psi(x)$ by

$$
\Psi(x)= \begin{cases}1, & |x| \leq 1 \\ \text { less than } 1, \text { positive, } & 1<|x|<2 \\ 0, & 2 \leq|x|\end{cases}
$$

and $\Psi_{R}(x)$ by

$$
\Psi_{R}(x)=\Psi\left(R^{-1} x\right) \quad\left(x \in \boldsymbol{R}^{2}, R>0\right)
$$

Put

$$
A_{R} \equiv\left\{x \in \boldsymbol{R}^{2} ; R \leq|x| \leq 2 R\right\}
$$

It follows from the definition of $\Psi_{R}$ that

$$
\operatorname{supp}\left[\nabla \Psi_{R}\right] \subset A_{R}, \quad \operatorname{supp}\left[\Delta \Psi_{R}\right] \subset A_{R} .
$$

Throughout this subsection we put

$$
H_{\text {int }}(t ; R)=\int_{\boldsymbol{R}^{2}}((1+u(t)) \log (1+u(t))-u(t)) \Psi_{R} d x-\frac{1}{2} \int_{\boldsymbol{R}^{2}} u(t) \psi_{m}(t) \Psi_{R} d x,
$$

where $\psi_{m}(t)=\psi(t)-[\psi(t)]_{B},[\psi(t)]_{B}=(1 /|B|) \int_{B} \psi(t) d x, B=B_{2 R}(0)$. 
Lemma 3.13. It holds that

$$
\begin{gathered}
\frac{d}{d t} H_{\text {int }}(t ; R)+\int_{\boldsymbol{R}^{2}} u(t)\left|\nabla \log (1+u(t))-\nabla \psi_{m}(t)\right|^{2} \Psi_{R} d x \\
\quad+\int_{\boldsymbol{R}^{2}}|\nabla \log (1+u(t))|^{2} \Psi_{R} d x \\
=\int_{\boldsymbol{R}^{2}} u(t) \log (1+u(t)) \Psi_{R} d x+F_{1}(t)+F_{2}(t),
\end{gathered}
$$

where

$$
\begin{aligned}
F_{1}(t)= & \int_{\boldsymbol{R}^{2}}((1+u(t)) \log (1+u(t))-u(t)) \Delta \Psi_{R} d x \\
& -\int_{\boldsymbol{R}^{2}} u(t) \psi_{m}(t) \nabla \psi_{m}(t) \cdot \nabla \Psi_{R} d x+\int_{\boldsymbol{R}^{2}} \psi_{m}(t) \nabla u(t) \cdot \nabla \Psi_{R} d x \\
& +\int_{\boldsymbol{R}^{2}}(u(t) \log (1+u(t))-\log (1+u(t))) \nabla \psi_{m}(t) \cdot \nabla \Psi_{R} d x \\
F_{2}(t)= & -\int_{\boldsymbol{R}^{2}} \partial_{t} \psi_{m}(t) \nabla \psi_{m}(t) \cdot \nabla \Psi_{R} d x-\frac{1}{4} \frac{d}{d t} \int_{\boldsymbol{R}^{2}}\left|\psi_{m}(t)\right|^{2} \Delta \Psi_{R} d x
\end{aligned}
$$

Proof. We replace $\Phi_{n}$ and $|\nabla u|$ in (3.7) by $\Psi_{R}$ and $|\nabla(1+u)|$, respectively, and integrate on $\boldsymbol{R}^{2}$. Then, by the relation

$$
\int_{\boldsymbol{R}^{2}} \frac{|\nabla(1+u(t))|^{2}}{1+u(t)} \Psi_{R} d x=\int_{\boldsymbol{R}^{2}}(1+u(t))|\nabla \log (1+u(t))|^{2} \Psi_{R} d x
$$

we have

$$
\begin{aligned}
\frac{d}{d t} \int_{\boldsymbol{R}^{2}}((1 & +u(t)) \log (1+u(t))-u(t)) \Psi_{R} d x \\
& +\int_{\boldsymbol{R}^{2}}(1+u(t))|\nabla \log (1+u(t))|^{2} \Psi_{R} d x \\
& -\int_{\boldsymbol{R}^{2}} u(t) \nabla \log (1+u(t)) \cdot \nabla \psi_{m}(t) \Psi_{R} d x \\
= & \int_{\boldsymbol{R}^{2}}((1+u(t)) \log (1+u(t))-u(t)) \Delta \Psi_{R} d x \\
& +\int_{\boldsymbol{R}^{2}} u(t) \log (1+u(t)) \nabla \psi_{m}(t) \cdot \nabla \Psi_{R} d x .
\end{aligned}
$$

Multiplying the equation of $u$ by $\psi_{m} \Psi_{R}$ and integrating on $\boldsymbol{R}^{2}$, by integration by parts we have 


$$
\begin{aligned}
\int_{\boldsymbol{R}^{2}} \partial_{t} u(t) \psi_{m}(t) \Psi_{R} d x= & -\int_{\boldsymbol{R}^{2}} \nabla u(t) \cdot \nabla \psi_{m}(t) \Psi_{R} d x \\
& -\int_{\boldsymbol{R}^{2}} \psi_{m}(t) \nabla u(t) \cdot \nabla \Psi_{R} d x \\
& +\int_{\boldsymbol{R}^{2}} u(t)\left|\nabla \psi_{m}(t)\right|^{2} \Psi_{R} d x \\
& +\int_{\boldsymbol{R}^{2}} \psi_{m}(t) u(t) \nabla \psi_{m}(t) \cdot \nabla \Psi_{R} d x
\end{aligned}
$$

Next, by $-\Delta \psi_{m}=u$,

$$
\begin{aligned}
\int_{\boldsymbol{R}^{2}} u \partial_{t} \psi_{m} \Psi_{R} d x & =-\int_{\boldsymbol{R}^{2}} \Delta \psi_{m} \partial_{t} \psi_{m} \Psi_{R} d x \\
& =\frac{1}{2} \frac{d}{d t} \int_{\boldsymbol{R}^{2}}\left|\nabla \psi_{m}\right|^{2} \Psi_{R} d x+\int_{\boldsymbol{R}^{2}} \partial_{t} \psi_{m} \nabla \psi_{m} \cdot \nabla \Psi_{R} d x
\end{aligned}
$$

Observing

$$
\begin{aligned}
\int_{\boldsymbol{R}^{2}}\left|\nabla \psi_{m}\right|^{2} \Psi_{R} d x & =-\int_{\boldsymbol{R}^{2}} \Delta \psi_{m} \psi_{m} \Psi_{R} d x-\int_{\boldsymbol{R}^{2}} \psi_{m} \nabla \psi_{m} \cdot \nabla \Psi_{R} d x \\
& =\int_{\boldsymbol{R}^{2}} u \psi_{m} \Psi_{R} d x+\frac{1}{2} \int_{\boldsymbol{R}^{2}} \psi_{m}^{2} \Delta \Psi_{R} d x
\end{aligned}
$$

and substituting this relation into (3.49), we obtain

$$
\begin{aligned}
\int_{\boldsymbol{R}^{2}} u \partial_{t} \psi_{m} \Psi_{R} d x= & \frac{1}{2} \frac{d}{d t} \int_{\boldsymbol{R}^{2}} u \psi_{m} \Psi_{R} d x+\frac{1}{4} \frac{d}{d t} \int_{\boldsymbol{R}^{2}} \psi_{m}^{2} \Delta \Psi_{R} d x \\
& +\int_{\boldsymbol{R}^{2}} \partial_{t} \psi_{m} \nabla \psi_{m} \cdot \nabla \Psi_{R} d x
\end{aligned}
$$

Adding (3.48) and (3.50) yields that

$$
\text { (3.51) } \begin{aligned}
\frac{1}{2} \frac{d}{d t} \int_{\boldsymbol{R}^{2}} u \psi_{m} \Psi_{R} d x= & -\int_{\boldsymbol{R}^{2}} \nabla u(t) \cdot \nabla \psi_{m}(t) \Psi_{R} d x+\int_{\boldsymbol{R}^{2}} u(t)\left|\nabla \psi_{m}(t)\right|^{2} \Psi_{R} d x \\
& -\int_{\boldsymbol{R}^{2}} \psi_{m}(t) \nabla u(t) \cdot \nabla \Psi_{R} d x \\
& +\int_{\boldsymbol{R}^{2}} u(t) \psi_{m}(t) \nabla \psi_{m}(t) \cdot \nabla \Psi_{R} d x \\
& +\int_{\boldsymbol{R}^{2}} \partial_{t} \psi_{m} \nabla \psi_{m} \cdot \nabla \Psi_{R} d x+\frac{1}{4} \frac{d}{d t} \int_{\boldsymbol{R}^{2}} \psi_{m}^{2} \Delta \Psi_{R} d x .
\end{aligned}
$$

It follows from (3.47) and (3.51) that 


$$
\begin{aligned}
& \frac{d}{d t} H_{\text {int }}(t ; R)+\int_{\boldsymbol{R}^{2}}|\nabla \log (1+u(t))|^{2} \Psi_{R} d x+I(t) \\
& =\int_{\boldsymbol{R}^{2}}((1+u(t)) \log (1+u(t))-u(t)) \Delta \Psi_{R} d x \\
& \quad+\int_{\boldsymbol{R}^{2}} u(t) \log (1+u(t)) \nabla \psi_{m}(t) \cdot \nabla \Psi_{R} d x \\
& \quad+\int_{\boldsymbol{R}^{2}} \psi_{m}(t) \nabla u(t) \cdot \nabla \Psi_{R} d x-\int_{\boldsymbol{R}^{2}} u(t) \psi_{m}(t) \nabla \psi_{m}(t) \cdot \nabla \Psi_{R} d x \\
& \quad-\int_{\boldsymbol{R}^{2}} \partial_{t} \psi_{m}(t) \nabla \psi_{m}(t) \cdot \nabla \Psi_{R} d x-\frac{1}{4} \frac{d}{d t} \int_{\boldsymbol{R}^{2}} \psi_{m}^{2} \Delta \Psi_{R} d x
\end{aligned}
$$

where

$$
\begin{aligned}
I(t)= & \int_{\boldsymbol{R}^{2}} u(t)|\nabla \log (1+u(t))|^{2} \Psi_{R} d x \\
& -\int_{\boldsymbol{R}^{2}} u(t) \nabla \psi_{m}(t) \cdot \nabla \log (1+u(t)) \Psi_{R} d x \\
& -\int_{\boldsymbol{R}^{2}} \nabla u(t) \cdot \nabla \psi_{m}(t) \Psi_{R} d x+\int_{\boldsymbol{R}^{2}} u(t)\left|\nabla \psi_{m}(t)\right|^{2} \Psi_{R} d x .
\end{aligned}
$$

We observe that

$$
\begin{aligned}
I(t)= & \int_{\boldsymbol{R}^{2}} u(t)\left|\nabla \log (1+u(t))-\nabla \psi_{m}(t)\right|^{2} \Psi_{R} d x \\
& -\int_{\boldsymbol{R}^{2}} \nabla \psi_{m}(t) \cdot \nabla \log (1+u(t)) \Psi_{R} d x,
\end{aligned}
$$

and that by integration by parts and $-\Delta \psi_{m}=u$,

$$
\begin{aligned}
-\int_{\boldsymbol{R}^{2}} \nabla \psi_{m} \cdot \nabla \log (1+u) \Psi_{R} d x= & -\int_{\boldsymbol{R}^{2}} u \log (1+u) \Psi_{R} d x \\
& +\int_{\boldsymbol{R}^{2}} \log (1+u) \nabla \psi_{m} \cdot \nabla \Psi_{R} d x
\end{aligned}
$$

Hence

$$
\begin{aligned}
I(t)= & \int_{\boldsymbol{R}^{2}} u(t)\left|\nabla \log (1+u(t))-\nabla \psi_{m}(t)\right|^{2} \Psi_{R} d x \\
& -\int_{\boldsymbol{R}^{2}} u \log (1+u) \Psi_{R} d x+\int_{\boldsymbol{R}^{2}} \log (1+u) \nabla \psi_{m} \cdot \nabla \Psi_{R} d x .
\end{aligned}
$$

Substituting this into (3.52) completes the proof of Lemma 3.13. 
In the rest of this subsection, we take $R=4 \tilde{R}_{0}$, where $\tilde{R}_{0}$ is the one determined in Proposition 3.12.

Lemma 3.14. For $F_{1}(t)$ in Lemma 3.13, it holds that

$$
\sup _{0<t<T}\left|F_{1}(t)\right|<\infty \text {. }
$$

Proof. Since the supports of $\nabla \Psi_{R}$ and $\Delta \Psi_{R}$ are in $A_{R}=\left\{x \in \boldsymbol{R}^{2} ; R \leq\right.$ $|x| \leq 2 R\}$, the integral regions appearing in $F_{1}(t)$ can be taken as $A_{R}$. The integrands in $F_{1}(t)$ are bounded on $[0, T) \times A_{R}$ by (1) and (2) of Proposition 3.12 , and hence we conclude the boundedness of $F_{1}(t)$ on $[0, T)$.

Lemma 3.15. For $F_{2}(t)$ in Lemma 3.13, it holds that

$$
\sup _{0<t<T}\left|\int_{0}^{t} F_{2}(s) d s\right|<\infty .
$$

Proof. Let $J_{R}$ be a function in $C_{0}^{\infty}\left(\boldsymbol{R}^{2}\right)$ such that

$$
\begin{aligned}
& J_{R}(x)= \begin{cases}1, & R \leq|x| \leq 2 R, \\
l e s s \text { than } 1, \text { positive, } & R / 2<|x|<R, 2 R<|x| \leq 3 R, \\
0, & \text { otherwise, }\end{cases} \\
& \left|\nabla J_{R}(x)\right| \leq C R^{-1}, \quad\left|\Delta J_{R}(x)\right| \leq C R^{-2},
\end{aligned}
$$

and put $\tilde{\Psi}_{R}(x)=\Psi_{R}(x) J_{R}(x)$. Since $\operatorname{supp}\left[\nabla \Psi_{R}\right] \subset A_{R}$, with $\tilde{\Psi}_{R}$ we can rewrite $F_{2}(t)$ as

$$
F_{2}(t)=-\int_{\boldsymbol{R}^{2}} \partial_{t} \psi_{m} \nabla \psi_{m} \cdot \nabla \tilde{\boldsymbol{\Psi}}_{R} d x-\frac{1}{4} \frac{d}{d t} \int_{\boldsymbol{R}^{2}} \psi_{m}^{2} \Delta \tilde{\boldsymbol{\Psi}}_{R} d x
$$

Noting

$$
\begin{aligned}
-\partial_{t} \psi_{m} \nabla \psi_{m} \cdot \nabla \tilde{\boldsymbol{\Psi}}_{R}= & -\nabla \cdot\left(\partial_{t} \psi_{m} \psi_{m} \nabla \tilde{\boldsymbol{\Psi}}_{R}-\left(\partial_{t} \nabla \psi_{m}\right) \psi_{m} \tilde{\boldsymbol{\Psi}}_{R}\right)-\left(\partial_{t} \Delta \psi_{m}\right) \psi_{m} \tilde{\boldsymbol{\Psi}}_{R} \\
& -\frac{1}{2} \partial_{t}\left|\nabla \psi_{m}\right|^{2} \tilde{\boldsymbol{\Psi}}_{R}+\frac{1}{2} \partial_{t} \psi_{m}^{2} \Delta \tilde{\boldsymbol{\Psi}}_{R}
\end{aligned}
$$

and using $-\partial_{t} \Delta \psi_{m}=\partial_{t} u=\Delta u-\nabla u \cdot \nabla \psi_{m}+u^{2}$, we can rewrite $F_{2}(t)$ as

$$
\begin{aligned}
F_{2}(t)= & \frac{1}{4} \frac{d}{d t}\left\{\int_{\boldsymbol{R}^{2}} \psi_{m}^{2} \Delta \tilde{\Psi}_{R} d x-2 \int_{\boldsymbol{R}^{2}}\left|\nabla \psi_{m}\right|^{2} \tilde{\boldsymbol{\Psi}}_{R} d x\right\} \\
& +\int_{\boldsymbol{R}^{2}}\left(\Delta u-\nabla u \cdot \nabla \psi_{m}+u^{2}\right) \psi_{m} \tilde{\boldsymbol{\Psi}}_{R} d x \\
= & F_{21}(t)+F_{22}(t) .
\end{aligned}
$$


Put $\tilde{A}_{R}=\left\{x \in \boldsymbol{R}^{2} ; R / 2 \leq|x| \leq 3 R\right\}$. Since the support of $\tilde{\boldsymbol{\Psi}}_{R}$ is included in $\tilde{A}_{R}$, by (2) of Proposition 3.12 we have

$$
\begin{aligned}
& \sup _{0<t<T} \int_{\boldsymbol{R}^{2}} \psi_{m}^{2}(t)|\Delta \tilde{\boldsymbol{\Psi}}| d x<\infty, \\
& \sup _{0<t<T} \int_{\boldsymbol{R}^{2}}\left|\nabla \psi_{m}(t)\right|^{2} \tilde{\boldsymbol{\Psi}}_{R} d x<\infty,
\end{aligned}
$$

and hence

$$
\sup _{0<t<T}\left|\int_{0}^{t} F_{21}(s) d s\right|<\infty .
$$

For $F_{22}(t)$, we rewrite $F_{22}(t)$ as

$$
F_{22}(t)=-2 \int_{\boldsymbol{R}^{2}} \nabla u \cdot \nabla \psi_{m} \tilde{\Psi}_{R} d x-\int_{\boldsymbol{R}^{2}} \nabla u \cdot \nabla \tilde{\Psi}_{R} \psi_{m} d x+\int_{\boldsymbol{R}^{2}} u^{2} \psi_{m} \tilde{\Psi}_{R} d x .
$$

Since the integrands in $F_{22}(t)$ are bounded on $(0, T) \times \tilde{A}_{R}$ by (1) and (2) of Proposition 3.12 , we also have

$$
\sup _{0<t<T}\left|F_{22}(t)\right|<\infty .
$$

Therefore Lemma 3.15 is established.

Proposition 3.16. Assume $\int_{\boldsymbol{R}^{2}} u_{0} d x \leq 8 \pi$. Then

$$
\sup _{0<t<T} \int_{|x| \leq 4 \tilde{R}_{0}}(1+u(t)) \log (1+u(t)) d x<\infty,
$$

where $\tilde{R}_{0}$ is the one determined in Proposition 3.12 .

Proof. Put $R=4 \tilde{R}_{0}$. It follows from Lemma 3.13 that

$$
\frac{d}{d t} H_{\text {int }}(t ; R) \leq \int_{\boldsymbol{R}^{2}}(1+u(t)) \log (1+u(t)) \Psi_{R} d x+F_{1}(t)+F_{2}(t) .
$$

For $0<a<1$,

$$
\frac{1}{2} u \psi_{m} \leq(1-a) u \frac{\left|\psi_{m}\right|}{2(1-a)} \leq(1-a)(1+u) \log (1+u)+(1-a) \exp \left\{\frac{\left|\psi_{m}\right|}{2(1-a)}\right\},
$$

from which it follows that

$$
(1+u) \log (1+u) \leq \frac{1}{a}\left((1+u) \log (1+u)-\frac{1}{2} u \psi_{m}\right)+\frac{1-a}{a} \exp \left\{\frac{\left|\psi_{m}\right|}{2(1-a)}\right\} .
$$


Then

$$
\begin{aligned}
\int_{\boldsymbol{R}^{2}}( & +u(t)) \log (1+u(t)) \Psi_{R} d x \\
& \leq \frac{1}{a} H_{\text {int }}(t ; R)+\frac{1}{a} \int_{\boldsymbol{R}^{2}} u(t) \Psi_{R} d x+\frac{1-a}{a} \int_{\boldsymbol{R}^{2}} \exp \left\{\frac{\left|\psi_{m}(t)\right|}{2(1-a)}\right\} \Psi_{R} d x \\
& \leq \frac{1}{a} H_{\text {int }}(t ; R)+\frac{1}{a}\left\|u_{0}\right\|_{1}+F_{3}(t),
\end{aligned}
$$

where

$$
F_{3}(t)=\frac{1-a}{a} \int_{R^{2}} \exp \left\{\frac{\left|\psi_{m}(t)\right|}{2(1-a)}\right\} \Psi_{R} d x .
$$

By (3.53) and (3.54) we have

$$
\frac{d}{d t} H_{\text {int }}(t ; R) \leq \frac{1}{a} H_{\text {int }}(t ; R)+F(t),
$$

where

$$
F(t)=F_{1}(t)+F_{2}(t)+F_{3}(t)+\frac{1}{a}\left\|u_{0}\right\|_{1} .
$$

We claim

$$
\sup _{0<t<T} F_{3}(t)<\infty .
$$

To prove this claim, by (3) of Proposition 3.12 we firstly note that there exist $x_{0} \in \boldsymbol{R}^{2}, \varepsilon_{0}>0, \delta>0$, satisfying $R+\varepsilon_{0}<\left|x_{0}\right|<2 R$, such that

$$
u(t, x) \geq \delta \quad \text { for } 0 \leq t<T,\left|x-x_{0}\right| \leq \varepsilon_{0} .
$$

By (2) of Proposition 3.12,

$$
M:=\sup _{0<t<T}\left\|\psi_{m}(t)\right\|_{L^{\infty}(R \leq|x| \leq 2 R)}<\infty .
$$

The function $\psi_{m}(t)$ satisfies

$$
-\Delta\left(\frac{\psi_{m}(t)}{2(1-a)}\right)=\frac{u(t)}{2(1-a)}, \quad|x|<R
$$

and $\sup _{|x|=R}\left|\psi_{m}(t)\right| \leq M(0<t<T)$. By (3.57), we have

$$
\begin{aligned}
\int_{|x| \leq R} u(t) d x & \leq \int_{\boldsymbol{R}^{2}} u(t) d x-\int_{\left|x-x_{0}\right| \leq \varepsilon_{0}} u(t) d x \\
& \leq 8 \pi-\delta \pi \varepsilon_{0}^{2}=\pi\left(8-\delta \varepsilon_{0}^{2}\right)
\end{aligned}
$$


and hence

$$
\int_{|x| \leq R} \frac{u(t)}{2(1-a)} d x \leq \frac{\pi}{2(1-a)}\left(8-\delta \varepsilon_{0}^{2}\right) .
$$

We choose $0<a<1$ such that

$$
\frac{\pi}{2(1-a)}\left(8-\delta \varepsilon_{0}^{2}\right)<4 \pi
$$

and apply Lemma 2.7 to get that for $0<t<T$,

$$
\begin{aligned}
\int_{|x|<R} \exp \left\{\frac{\left|\psi_{m}(t)\right|}{2(1-a)}\right\} d x & \leq \frac{32 \pi(1-a) R^{2}}{\delta \varepsilon_{0}^{2}-8 a} \exp \left\{\sup _{|x|=R} \frac{\left|\psi_{m}(t)\right|}{2(1-a)}\right\} \\
& \leq \frac{32 \pi(1-a) R^{2}}{\delta \varepsilon_{0}^{2}-8 a} \exp \left\{\frac{M}{2(1-a)}\right\}
\end{aligned}
$$

Hence

$$
\int_{R^{2}} \exp \left\{\frac{\left|\psi_{m}(t)\right|}{2(1-a)}\right\} \Psi_{R} d x \leq\left(\frac{32 \pi(1-a) R^{2}}{\delta \varepsilon_{0}^{2}-8 a}+3 \pi R^{2}\right) \exp \left\{\frac{M}{2(1-a)}\right\},
$$

which implies (3.56).

By (3.55), we have

$$
H_{\text {int }}(t ; R) \leq H_{\text {int }}(0 ; R)+\frac{1}{a} \int_{0}^{t} H_{\text {int }}(s ; R) d s+\sup _{0<t<T}\left|\int_{0}^{t} F(s) d s\right|, \quad 0<t<T
$$

and by Lemmas 3.14 and 3.15 and (3.56),

$$
\sup _{0<t<T}\left|\int_{0}^{t} F(s) d s\right|<\infty .
$$

Applying the Gronwall inequality, we deduce that

$$
\sup _{0<t<T} H_{\text {int }}(t ; R)<\infty .
$$

Therefore, by this estimate, (3.54) and (3.56), we obtain

$$
\sup _{0<t<T} \int_{\boldsymbol{R}^{2}}(1+u(t)) \log (1+u(t)) \Psi_{R} d x<\infty,
$$

which completes the proof of Proposition 3.16.

Acknowledgement. We are very grateful to Professor Noriko Mizoguchi for sending us her preprints on the global existence of nonnegative solutions 
to the Cauchy problem for the parabolic system (1.2) in $\boldsymbol{R}^{2}$. The work of T. Ogawa is partially supported by JSPS Grant-in-Aid for Scientific Research A \#20244009 and S \#24220702.

\section{References}

[1] Ben-Artzi, M., Global solutions of two-dimensional Navier-Stokes and Euler equations, Arch. Rational Mech. Anal., 128 (1994), 329-358.

[2 ] Benilan, P., Brezis, H. and Crandall, M., A semilinear equation in $L^{1}\left(\boldsymbol{R}^{N}\right)$, Ann. Scuola Norm. Sup. Pisa Cl. Sci. (4), 2 (1975), 523-555.

[3] Biler, P., Existence and nonexistence of solutions for a model of gravitational interaction of particles, III, Colloq. Math., 68 (1995), 229-239.

[4] Biler, P., Local and global solvability of some parabolic systems modelling chemotaxis, Adv. Math. Sci. Appl., 8 (1998), 715-743.

[ 5] Biler, P. and Dolbeault, J., Long time behavior of solutions to Nernst-Planck and DebyeHückel drift-diffusion systems, Ann. Henri Poincaré, 1 (2000), 461-472.

[6] Biler, P., Hilhorst, D. and Nadzieja, T., Existence and nonexistence of solutions for a model of gravitational interaction of particles, II, Colloq. Math., 67 (1994), 297-308.

[ 7 ] Biler, P., Cannone, M., Guerra, I. A. and Karch, G., Global regular and singular solutions for a model of gravitating particles, Math. Ann., 330 (2004), 693-708.

[ 8 ] Biler, P., Karch, G., Laurençot, Ph. and Nadzieja, T., The $8 \pi$-problem for radially symmetric solutions of a chemotaxis model in the plane, Math. Methods Appl. Sci., 29 (2006), 15631583.

[9] Biler, P. and Nadzieja, T., Existence and nonexistence of solutions for a model of gravitational interaction of particles I, Colloq. Math., 66 (1994), 319-334.

[10] Biler, P. and Nadzieja, T., A nonlocal singular parabolic problem modelling gravitational interaction of particles, Adv. Differential Equations, 3 (1998), 177-197.

[11] Blanchet, A., Carlen, E. and Carrillo, J. A., Functional inequalities, thick tails and asymptotics for the critical mass Patlak-Keller-Segel model, J. Funct. Anal., 262 (2012), 2142-2230.

[12] Blanchet, A., Carrillo, J. A. and Laurençot, P., Critical mass for a Patlak-Keller-Segel model with degenerate diffusion in higher dimensions, Calc. Var. Partial Differential Equations, 35 (2009), 133-168.

[13] Blanchet, A., Carrillo, J. A. and Masmoudi, N., Infinite time aggregation for the critical Patlak-Keller-Segel model in $\boldsymbol{R}^{2}$, Comm. Pure Appl. Math., 61 (2008), 1449-1481.

[14] Blanchet, A., Dolbeault, J. and Perthame, B., Two-dimensional Keller-Segel model: optimal critical mass and qualitative properties of the solutions, Electron. J. Differential Equations, 2006 (2006), No. 44, 33pp.

[15] Brezis, H., Remarks on the preceding paper by M. Ben-Artzi: Global solutions of twodimensional Navier-Stokes and Euler equations, Arch. Rational Mech. Anal., 128 (1994), 359-360.

[16] Brezis, H. and Merle, F., Uniform estimate and blow-up behavior for solutions of $-\Delta u=$ $V(x) e^{u}$ in two dimensions, Comm. Partial Differential Equations, 16 (1991), 1223-1253.

[17] Calvez, V. and Corrias, L., The parabolic-parabolic Keller-Segel model in $\boldsymbol{R}^{2}$, Commun. Math. Sci., 6 (2008), 417-447.

[18] Childress, S. and Percus, J. K., Nonlinear aspects of chemotaxis, Math. Biosci., 56 (1981), 217-237. 
[19] Gajewski, H. and Zacharias, K., Global behaviour of a reaction-diffusion system modelling chemotaxis, Math. Nachr., 195 (1998), 77-114.

[20] Giga, Y., Solutions for semilinear Parabolic equations in $L^{p}$ and regularity of weak solutions of the Navier-Stokes system, J. Differential Equations, 61 (1986), 186-212.

[21] Giga, Y. and Kambe, T., Large time behavior of the vorticity of two-dimensional viscous flow and its application to vortex formation, Comm. Math. Phys., 117 (1988), 549-568.

[22] Giga, Y. and Miyakawa, T., Solutions in $L^{r}$ of the Navier-Stokes initial value problem, Arch. Rational Mech. Anal., 89 (1985), 267-281.

[23] Giga, Y., Miyakawa, T. and Osada, H., Two-dimensional Navier-Stokes flow with measure as initial vorticity, Arch. Rational Mech. Anal., 104 (1988), 223-250.

[24] Gilberg, D. and Trudinger, S., Elliptic Partial Differential Equations of Second Order, Second Edition, Grundlehren der Mathematischen Wissenschaften, 224, Springer-Verlag, Berlin 1983.

[25] Herrero, M. A. and Velázquez, J. J. L., Singularity patterns in a chemotaxis model, Math. Ann., 306 (1996), 583-623.

[26] Herrero, M. A. and Velázquez, J. J. L., A blow-up mechanism for a chemotaxis model, Ann. Scuola Norm. Sup. Pisa Cl. Sci. (4), 24 (1997), 633-683.

[27] Horstmann, D., From 1970 until present: The Keller-Segel model in chemotaxis and its consequences, I, Jahresber. Deutsch. Math.-Verein., 105 (2003), 103-165.

[28] Horstmann, D. and Wang, G., Blow-up in a chemotaxis model without symmetry assumptions, European J. Appl. Math., 12 (2001), 159-177.

[29] Jäger, W. and Luckhaus, S., On explosions of solutions to a system of partial differential equations modelling chemotaxis, Trans. Amer. Math. Soc., 329 (1992), 819-824.

[30] Kato, T., The Navier-Stokes equation for an incompressible fluid in $R^{2}$ with a measure as the initial vorticity, Differential Integral Equations, 7 (1994), 949-966.

[31] Keller, E. F. and Segel, L. A., Initiation of slime mold aggregation viewed as an instability, J. Theoret. Biol., 26 (1970), 399-415.

[32] Kimijima, T., Nakagawa, K. and Ogawa, T., Threshold of global behavior of solutions to a degenerate drift-diffusion system in between two critical exponents, Calc. Var. Partial Differential Equations, 53 (2015), 441-472.

[33] Kobayashi, T. and Ogawa, T., Fluid Mechanical Approximation to the Degenerated DriftDiffusion System from the Compressible Navier-Stokes-Poisson System, Indiana Univ. Math. J., 62 (2013), 1021-1054.

[34] Kurokiba, M. and Ogawa, T., Finite time blow-up of the solution for a nonlinear parabolic equation of drift-diffusion type, Differential Integral Equations, 16 (2003), 427-452.

[35] Kurokiba, M. and Ogawa, T., Well-posedness of the for the drift-diffusion system in $L^{p}$ arising from the semiconductor device simulation, J. Math. Anal. Appl., 342 (2008), 10521067.

[36] Kurokiba, M. and Ogawa, T., Two dimensional drift-diffusion system in a critical weighted space, Differential Integral Equations, 28 (2015), 753-776.

[37] Kurokiba, M. and Ogawa, T., Finite time blow up for a solution to system of the driftdiffusion equations in higher dimensions, preprint.

[38] Ladyzenskaja, O. A., Solonnikov, V. A. and Ural'ceva, N. N., Linear and Quasi-linear Equations of Parabolic Type, Translations of Mathematical Monographs, Vol. 23, American Mathematical Society, Providence, R.I., 1968.

[39] López-Gómez, J., Nagai, T. and Yamada, T., The basin of attraction of the steady-states for a chemotaxis model in $\boldsymbol{R}^{2}$ with critical mass, Arch. Ration. Mech. Anal., 207 (2013), 159-184. 
[40] Mizoguchi, N., Global existence for the Cauchy problem of the parabolic-parabolic KellerSegel system on the plane, Calc. Var. Partial Differential Equations, 48 (2013), 491-505.

[41] Mock, M. S., An initial value problem from semiconductor device theory, SIAM J. Math. Anal., 5 (1974), 597-612.

[42] Nagai, T., Blow-up of radially symmetric solutions to a chemotaxis system, Adv. Math. Sci. Appl., 5 (1995), 581-601.

[43] Nagai, T., Blowup of nonradial solutions to parabolic-elliptic systems modeling chemotaxis in two-dimensional domains, J. Inequal. Appl., 6 (2001), 37-55.

[44] Nagai, T., Global solvability for a chemotaxis system in $\boldsymbol{R}^{2}$, RIMS Kôkyûroku Bessatsu, B15, Res. Inst. Math. Sci. (RIMS), Kyoto, 2009, pp. 101-111.

[45] Nagai, T., Global existence and decay estimates of solutions to a parabolic-elliptic system of drift-diffusion type in $\boldsymbol{R}^{2}$, Differential Integral Equations, 24 (2011), 29-68.

[46] Nagai, T. and Ogawa, T., Brezis-Merle inequalities and application to the global existence of the Cauchy problem of the Keller-Segel system, Commun. Contemp. Math., 13 (2011), 795-812.

[47] Nagai, T., Senba, T. and Suzuki, T., Chemotactic collapse in a parabolic system of mathematical biology, Hiroshima Math. J., 30 (2000), 463-497.

[48] Nagai, T., Senba, T. and Yoshida, K., Application of the Trudinger-Moser inequality to a parabolic system of chemotaxis, Funkcial. Ekvac., 40 (1997), 411-433.

[49] Nash, J., Continuity of solutions of parabolic and elliptic equations, Amer. J. Math., 80 (1958), 931-954.

[50] Ogawa, T., Asymptotic stability of a decaying solution to the Keller-Segel system of degenerate type, Differential Integral Equations, 21 (2008), 1113-1154.

[51] Ogawa, T., The degenerate drift-diffusion system with the Sobolev critical exponent, Discrete Contin. Dyn. Syst. Ser. S., 4 (2011), 875-886.

[52] Ogawa, T. and Shimizu, S., The drift-diffusion system in two dimensional critical Hardy space, J. Funct. Anal., 255 (2008) 1107-1138.

[53] Ogawa, T. and Shimizu, S., End-point maximal regularity and its application to twodimensional Keller-Segel system, Math. Z., 264 (2010), 601-628.

[54] Ogawa, T. and Wakui, H., Non-uniform bound and finite time blow up for solutions to a drift-diffusion equation in higher dimensions, Anal. Appl. (Singap.), 14 (2016), 145-183.

[55] Patlak, C. S., Random walk with persistence and external bias, Bull. Math. Biophys., 15 (1953), 311-338.

[56] Senba, T., Grow-up rate of a radial solution for a parabolic-elliptic system in $\boldsymbol{R}^{2}$, Adv. Differential Equations, 14 (2009), 1155-1192.

[57] Senba, T., Blowup in infinite time of radial solutions to parabolic-elliptic system in highdimensional Euclidean spaces, Nonlinear Anal., 70 (2009), 2549-2562.

[58] Senba, T. and Suzuki, T., Chemotactic collapse in a parabolic-elliptic system of mathematical biology, Adv. Differential Equations, 6 (2001), 21-50.

[59] Senba, T. and Suzuki, T., Blow up behavior of solutions to the rescaled Jäger-Luckhaus system, Adv. Differential Equations, 8 (2003), 787-820.

[60] Sugiyama, Y., Global existence in sub-critical cases and finite time blow-up in super-critical cases to degenerate Keller-Segel system, Differential Integral Equations, 19 (2006), 841-876.

[61] Suzuki, T., Free Energy and Self-Interacting Particles, Progress in Nonlinear Differential Equations and their Applications, 62, Birkhäuser Boston Inc., Boston, MA, 2005.

[62] Suzuki, T. and Takahashi, R., Degenerate parabolic equations with critical exponent derived from the kinetic theory II, Blowup threshold, Differential Integral Equations, 22 (2009), 1153-1172. 
[63] Stein, E., Harmonic Analysis, Princeton Mathematical Series, 43, Monographs in Harmonic Analysis, III, Princeton University Press, Princeton, NJ, 1993.

[64] Wolansky, G., Comparison between two models of self-gravitating clusters: conditions for gravitational collapse, Nonlinear Anal., 24 (1995), 1119-1129.

\author{
nuna adreso: \\ Toshitaka Nagai \\ Department of Mathematics \\ Graduate School of Science \\ Hiroshima University \\ 1-3-1 Kagamiyama, Higashi-Hiroshima \\ Hiroshima 739-8526 \\ Japan \\ Takayoshi Ogawa \\ Mathematical Institute \\ Tohoku University \\ 6-3, Aramaki Aza-Aoba, Aoba-ku, Sendai \\ Miyagi 980-8578 \\ Japan
}

(Ricevita la 23-an de aprilo, 2014)

(Reviziita la 20-an de aŭgusto, 2015) 